\title{
Phytohormone profiles are strongly altered during induction and symptom development of the physiological ripening disorder berry shrivel in grapevine
}

\author{
Michaela Griesser $^{1}$ (D) $\cdot$ Stefania Savoi ${ }^{2} \cdot$ Suriyan Supapvanich ${ }^{3} \cdot$ Petre Dobrev $^{4} \cdot$ Radomira Vankova $^{4} \cdot$ Astrid Forneck $^{1}$
}

Received: 18 October 2019 / Accepted: 13 February 2020 / Published online: 18 February 2020

(c) The Author(s) 2020

\begin{abstract}
The process of grape berry ripening follows three phases with distinct metabolic processes and complex regulations via phytohormones. The physiological ripening disorder berry shrivel (BS) is characterized by reduced sugar accumulation, low anthocyanin contents, and high acidity in affected berries. The processes leading to BS induction are unknown, but recent transcriptional data on reduced expression of switch genes hint towards a disturbed ripening onset. Herein we investigated the phytohormone composition throughout grape berry ripening in healthy and BS berries in Vitis vinifera L. cultivar Blauer Zweigelt. Thereby we hypothesize that phytohormones are key players for BS induction and suppress the expression of switch genes at veraison. The presented metabolomics and RNAseq data describe two distinct phytohormone profiles in BS berries, differing between pre- and post-veraison with a clear ethylene precursor (aminocyclopropane-1-carboxylic acid, ACC) peak before veraison. Exogenous application of ACC led to BS symptoms, while ethephone application led to berry abscission. During post-veraison, we observed high ABA-glucose ester (ABA-GE) and low indole-3-acetate aspartate (IAA-Asp) and isopentenyladenine (iP) contents in BS berries and the transcriptional induction of several phytohormone pathways. The presented descriptive data provide valuable knowledge to further decipher the role of phytohormones in BS induction and BS symptom development.
\end{abstract}

\section{Key message}

Ripening disorder berry shrivel in grapevine leads to distinct phytohormone pattern pre- and post-veraison in grape berries.

Keywords Grapevine $\cdot$ Ripening regulation $\cdot$ Physiological disorders $\cdot$ Phytohormones

Electronic supplementary material The online version of this article (https://doi.org/10.1007/s11103-020-00980-6) contains supplementary material, which is available to authorized users.

Michaela Griesser

michaela.griesser@boku.ac.at

1 Department of Crop Sciences, Institute of Viticulture and Pomology, University of Natural Resources and Life Sciences, Konrad Lorenz Straße 24, Tulln, 3430 Vienna, Austria

2 AGAP, Montpellier University, CIRAD, INRA, Montpellier SupAgro, 2 Place Pierre Viala, 34060 Montpellier, France

3 Department of Agricultural Education, Faculty of Industrial Education and Technology, King Mongkut's Institute of Technology Ladkrabang, 1 Chalongkrung Road, Ladkrabang, Bangkok 10520, Thailand

4 Institute of Experimental Botany, Czech Academy of Sciences, Prague, Czech Republic

\section{Introduction}

Sugar accumulation disorder, known as berry shrivel (BS), is a grapevine ripening disorder with still unclear physiological mechanisms. Grape berry quality and yield are highly affected by this disorder and BS grape clusters are not considered for winemaking. All shriveling disorders in grapevine like sunburn, late-season dehydration, bunch stem necrosis, and BS have the common feature of shrinking berries, but the time of emergence, the morpho-anatomical characteristics and the metabolite content are distinct from each other (Bondada and Keller 2012a; Griesser et al. 2012; Krasnow et al. 2010). Recognizable symptoms of BS are: a stop of sugar accumulation short after veraison, high contents of organic acids, low $\mathrm{pH}$ values and low amounts of anthocyanins in red grape varieties 
(Griesser et al. 2018; Savoi et al. 2019). Dissimilar from bunch stem necrosis, rachis and pedicels stay viable and green with no obvious symptoms on the surface. Nevertheless morpho-anatomical study observed cell death in the rachis of symptomatic grape clusters of Cabernet Sauvignon (Bondada and Keller 2012b; Hall et al. 2011), TEM analysis showed collapsed cells and cell wall thickenings in the secondary phloem (Crespo-Martinez et al. 2019) and light microscopy and SEM analyses revealed higher rates of callose deposition on sieve plates (Bondada 2016; Crespo-Martinez et al. 2019). Reduced cell viability was also observed in grape berries leading to the assumption that the loss of cell membrane integrity is an important factor for water loss and berry shrinkage (Krasnow et al. $2008,2012)$. The major drawback of these studies is the use of already symptomatic grape clusters, which prevents distinction of causal BS inducing events from the follow up symptoms. Recently, it was shown that transcriptional changes occur in BS grape berries at the onset of ripening before symptoms are visible, while no alterations in berry transcriptome were observed in pre-veraison samples (Griesser et al. 2017; Savoi et al. 2019). Specifically, a subset of genes, called switch genes, described as master regulators of the ripening onset in grape berries (Massonnet et al. 2017; Palumbo et al. 2014), were down-regulated in BS berries at veraison, suggesting an altered ripening induction (Savoi et al. 2019). Later ripening stages showed more than 3000 genes differentially expressed, among them up-regulation of genes related to phytohormone biosynthesis, response to stress and phenylpropanoid pathway, whereas genes related to the flavonoid pathway and the sugar metabolism were expressed to a lower extend in BS grapes (Savoi et al. 2019). In conclusion, low anthocyanin and sugar content are likely the consequences of a disturbance of the entire ripening process as indicated by the lower expression of several switch genes at veraison in BS berries. Phytohormone metabolites are possible candidates for switch gene regulation and many of the switch genes are assumed to be regulated by abscisic acid (ABA) (Pilati et al. 2017; Savoi et al. 2019).

The process of grape berry development and ripening displays a double sigmoid growth curve with three distinct phases (Coombe and Hale 1973). Veraison marks the initiation of sugar accumulation and the rapid pigmentation of berries by anthocyanins in red grape varieties. Grape berry ripening, especially the transition from the first growth phase to ripening related processes later in development, is very complex and just recently this complexity was revealed by applying omics techniques (Fasoli et al. 2018; Pilati et al. 2017). In general, it is assumed that ABA, ethylene, and brassinosteroids provide the signals for the onset of ripening although the processes are unclear as previously reviewed (Fortes et al. 2015; Kuhn et al. 2014b). Especially the role of ethylene in combination with other phytohormones is under debate (Chervin et al. 2006; Sun et al. 2010). Additionally, physical modifications prior to the ABA signal start the softening process of berries via loss of cell turgor and elasticity (Castellarin et al. 2016). Yet not fully understood, it is possible that an interplay takes place between ethylene and ABA during the ripening phase (Sun et al. 2010). Auxin is a negative regulator of berry ripening, as high contents are observed in green fast growing berries whereas very low levels are detected at veraison and throughout the ripening period (Bottcher et al. 2010). Exogenous artificial auxin application delayed ripening (Bottcher et al. 2011), along with a lower expression of genes related to the ABA biosynthesis and signaling pathways, while ethylene biosynthesis was induced (Ziliotto et al. 2012).

Apart from metabolic signals, a timeline of events leading to the onset of ripening was suggested with an initial fall of berry elasticity and turgor pressure followed by $\mathrm{ABA}$ and sugar accumulation and coloring pigments in skins of red grape varieties (Castellarin et al. 2016). More specifically two rapid transcriptional transitions starting 14 $\mathrm{d}$ before veraison have been characterized in grape berries, suggesting a hierarchy of signals for the onset of ripening (Fasoli et al. 2018). BS berries have low sugar contents and reduced anthocyanin biosynthesis (Griesser et al. 2018), suggesting a disturbed ripening process, which is supported by the transcriptional repression of switch genes in BS berries at veraison (Savoi et al. 2019). The phytohormone crosstalk regulating grape berry ripening is not fully understood, but there is a strong evidence that ABA, brassinosteroids and ethylene induce and promote ripening, while auxin can delay the major ripening associated processes. Here we firstly describe the phytohormone composition and linked gene expression of healthy and BS-grapes during the ripening period of two vintages on $V$. vinifera $\mathrm{cv}$. Zweigelt. We hypothesize that ethylene and its crosstalk with other hormones induces BS in grape berries. In general, ripening control by phytohormones needs further attention, like timing, sensitivity of the tissue, molecules involved and their concentrations.

\section{Materials and methods}

\section{Plant material and sampling}

Berry samples of the red grape cultivar Zweigelt ( $V$. vinifera), grafted on rootstock Kober 5BB, were collected in 2011 and 2013 from a commercial vineyard located in Lower Austria (Antlasberg, Mailberg GPS coordinates 48.6667, 16.1833; yearly climate conditions are shown in Figure S1) as previously described (Griesser et al. 2018, 
2017; Savoi et al. 2019). In both years, grape bunches were randomly labeled after anthesis and samples were obtained in 2011 from EL33 (BBCH79) till EL38 (BBCH89) and in 2013 from EL32 (BBCH77) till EL37 (BBCH89). This corresponds to 30-75 days after anthesis (DAA) as shown in Fig. 1. Samples were obtained from each labeled cluster once to allow an adequate a posteriori categorization into healthy $(\mathrm{H}$, control) or BS-affected (BS) ones by not influencing the ripening process. Sugar accumulation and anthocyanin biosynthesis have been described for $\mathrm{H}$ and BS samples elsewhere (Griesser et al. 2018; Savoi et al. 2019). Three biological replicates for each sampling point and condition were considered for all analyses conducted. Each biological replicate resulted from the pooling of three different bottom clusters and only samples which were clearly categorized into the categories "healthy, control" and "BS" were used for further analyses. BS is affecting whole clusters in case of Zweigelt, therefore the obtained samples are representative for the cluster. Before analyses, frozen berries were ground to a fine powder under liquid nitrogen using a ball mill (Retsch MM400).

\section{Phytohormone quantification}

Four sampling points $(42,55,68,75$ DAA corresponding to EL33, EL35, EL36/2, and EL37) from 2011 were selected to determine phytohormones in whole berries according to a method previously described (Dobrev and Kaminek 2002; Dobrev and Vankova 2012). In short, approximately $50 \mathrm{mg}$ (FW) samples were homogenized and extracted with cold $\left(-20{ }^{\circ} \mathrm{C}\right)$ methanol/water/formic acid (15/4/1, v/v/v). To account for sample losses and for quantification by isotope dilution, the following isotope-labelled internal standards (10 pmol/sample) were added: ${ }^{13} \mathrm{C}_{6}$-IAA (Cambridge Isotope Laboratories, Tewksbury, MA, USA), ${ }^{2} \mathrm{H}_{4}$-SA (Sigma Aldrich, St. Louis, MO, USA), ${ }^{2} \mathrm{H}_{5}$-JA (Olchemim, Olomouc, Czech Republic), ${ }^{2} \mathrm{H}_{3}$-PA, ${ }^{2} \mathrm{H}_{3}$-DPA (NRC-PBI, Saskatoon, Canada), ${ }^{2} \mathrm{H}_{6}$-ABA, ${ }^{2} \mathrm{H}_{5}-\mathrm{JA},{ }^{2} \mathrm{H}_{2}-\mathrm{GA}_{4},{ }^{2} \mathrm{H}_{5}$-trans $\mathrm{Z}$,
${ }^{2} \mathrm{H}_{5}$-transZR, ${ }^{2} \mathrm{H} 5$-transZ7G, ${ }^{2} \mathrm{H}_{5}$-transZ9G, ${ }^{2} \mathrm{H}_{5}$-trans $\mathrm{ZOG}$, ${ }^{2} \mathrm{H}_{5}$-transZROG, ${ }^{2} \mathrm{H}_{5}$-transZRMP, ${ }^{2} \mathrm{H}_{3}$-DZ, ${ }^{2} \mathrm{H}_{3}$-DZR, ${ }^{2} \mathrm{H}_{3}$ DZ9G, ${ }^{2} \mathrm{H}_{6}$-iP, ${ }^{2} \mathrm{H}_{6}$-iPR, ${ }^{2} \mathrm{H}_{6}$-iP7G, ${ }^{2} \mathrm{H}_{6}$-iP9G, ${ }^{2} \mathrm{H}_{6}$-iPRMP (Olchemim, Olomouc, Czech Republic). The extract was applied to a mixed mode reverse phase-cation exchange SPE column (Oasis-MCX, Waters, Milford, MA, USA). Hormone fraction eluted with methanol contained hormones of acidic character [auxins, ABA, salicylic acid (SA), jasmonic acid (JA), gibberellins (GA)]. The basic fraction was eluted with $0.35 \mathrm{M} \mathrm{NH}_{4} \mathrm{OH}$ in $60 \%$ methanol (cytokinins, ACC). Fractions were evaporated to dryness in a vacuum concentrator and dissolved into $30 \mu \mathrm{L}$ of $10 \%$ methanol. An aliquot $(10 \mu \mathrm{L})$ was analyzed using a high-performance liquid chromatography (HPLC) (Ultimate, 3000 Dionex, Sunnyvale, USA) coupled to a hybrid triple quadrupole/linear ion trap mass spectrometer (3200 Q TRAP, Applied Biosystems, Foster City, USA) set in selected reaction monitoring mode. Chromatographic conditions included a HPLC column Luna C18(2) $(100 \times 2 \mathrm{~mm}, 3 \mu \mathrm{m}$, Phenomenex, Torrance, USA) at

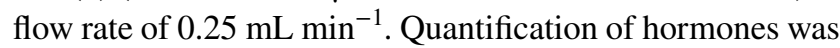
done using the isotope dilution method with multilevel calibration curves $\left(r^{2}>0.99\right)$. Data processing was carried out with Analyst 1.5 software (Applied Biosystems, Foster City, USA). Final compound determination values were given as absolute concentrations in $\mathrm{pmol} \mathrm{g}^{-1} \mathrm{DW}$.

\section{RNA extraction and expression analyses}

Total RNA from samples 2011 for qPCR analyses was extracted following the protocol previously described (Griesser et al. 2017; Reid et al. 2006), while RNA extraction from samples 2013 was performed with the 'Spectrum Plant total RNA' kit (Sigma-Aldrich) for RNASeq analyses.

The following chemicals were used for qPCR analyses: Amplification Grade DNase 1 (Sigma Aldrich) for DNA digestion, GoScript Reverse Transcription System (Promega, Madison, WI, USA) for cDNA synthesis, 2X KAPA

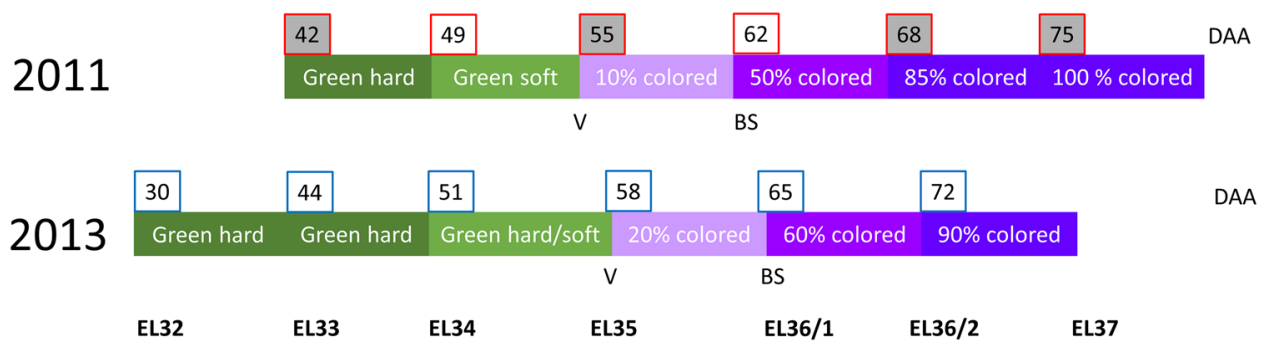

Fig. 1 Sampling scheme for 2011 and 2013 with observed phenology, EL categorization and days after anthesis (DAA). Grape berry development and ripening were slightly different between years but substantial overlaps were obtained for comparison. Samples for phytohormone and qPCR analyses (grey background) were obtained at
EL33, EL35, EL36/2 and EL37 corresponding to 42, 55, 68 and 75 DAA collected 2011. RNA Seq analyses and qPCR were performed using samples from 2013. Veraison (V) is stated as the onset of coloring and the first BS symptoms were observed at EL36/1 in both years 
SYBR FAST qPCR Universal (Peqlab, Erlangen, Germany) for qPCRs. Cycling conditions were as follows: activation 4 min at $95{ }^{\circ} \mathrm{C}, 40$ cycles for $8 \mathrm{~s}$ at $95^{\circ} \mathrm{C}, 20 \mathrm{~s}$ at $60^{\circ} \mathrm{C}, 30 \mathrm{~s}$ at $72{ }^{\circ} \mathrm{C}$ and $5 \mathrm{~s}$ at $75{ }^{\circ} \mathrm{C}$ with fluorescence measurement. The reference genes actin (VIT_04s0044g00580) and ef1 (VIT_06s0004g03220) were used for qPCR and data were calculated as NRQ (Hellemans et al. 2007) with the R-package "EasyqpcR". List of primers used is shown in Table S1.

\section{Induction of BS by exogenous ethylene application}

The phytohormone quantification showed a strong increase of ACC in BS berries before veraison. The effect of exogenous ethylene application on BS incidence was tested by applying four different treatments: control (water spraying), $1500 \mathrm{mg} \mathrm{L}^{-1}$ Ethephon (2-chloroethylphosphonic acid, Bayer Crop Science), $1000 \mathrm{mg} \mathrm{L}^{-1}$ ACC (1-aminocyclopropane-1-carboxylic acid, VBC-30426 Testproduct (10\%), Valent BioScience), and $250 \mathrm{mg} \mathrm{L}^{-1}$ AVG (L- $\alpha-(2-$ aminoethoxyvinyl)glycine hydrochloride, ReTain (15\%), Valent BioScience). Chemicals were applied to Zweigelt grape clusters (clone St.9 grafted on Kober 5BB, planted 2011 UFT experimental vineyard, Tulln, Lower Austria). The spraying was performed once with $10 \mathrm{~mL}$ solution each (30 grapes per treatment) on the 22.07.2016, 10 days before veraison. All grape clusters were evaluated on the 13.09.2016.

\section{Statistical analysis}

All statistical comparisons were conducted using IBM SPSS Statistics 21. Normal distribution of data sets was tested using the Shapiro-Wilk test. Significant differences were tested by comparison of healthy and BS affected berries with Student's $t$ test $(P<0.05)$ if normal distribution was ensured, otherwise non-parametric Mann-Whitney-U tests were conducted. Heatmaps were drawn using R.

\section{Results and discussion}

The phytohormone profile (metabolite levels and gene expression) of berries from the red grape cultivar Zweigelt in healthy grape clusters $(\mathrm{H})$ and grape clusters developing BS symptoms, a physiological ripening disorder, was analyzed from pre-veraison (pre-symptomatic grape clusters, EL32, EL33, EL34), veraison (EL35) until ripening (with and without BS symptoms, EL36/2, EL37). In total, 45 phytohormone metabolites were detected and quantified at four time points (Table 1). Additionally the transcriptional regulations of phytohormone related genes in $\mathrm{H}$ and BS berries obtained from RNASeq analyses (Savoi et al.
2019) are shown as heatmaps and have been validated with qPCR.

\section{Major induction of ABA pathways during the ripening phase and minor reduction of $A B A$ at veraison in $B S$ berries}

Abscisic acid (ABA) and its three metabolites ABA glucose ester (ABA-GE), phaseic acid (PA), and dihydrophaseic acid (DPA) were quantified in $\mathrm{H}$ and BS berries (Table 1). Our data (Fig. 2a) show an increase of ABA at veraison (EL35) in both $\mathrm{H}$ and BS berries and its decrease during the ripening phase (EL37). These results are in accordance with previous studies (Castellarin et al. 2016; Gambetta et al. 2010; Wheeler et al. 2009). An activation of ABA biosynthesis genes before veraison that could explain the ABA peak at veraison is not observed (Fig. 2g). In accordance, high ABA content without the expression of ABA biosynthesis genes have also been described in grape berries at softening and at veraison (Castellarin et al. 2016; Coelho et al. 2019). A cultivar-dependency was observed and it was proposed that the ABA increase at veraison might be caused by ABA import from other tissues or its lower turnover, which is supported by a decreased expression of ABA 8'-hydroxylases (Coelho et al. 2019). In BS-berries, the ABA content was slightly lower until veraison and higher thereafter (no significant values). This trend was in accordance with a strong increase in expression of VviNCED2 in BS berries starting from EL35 till EL37 (Fig. 2d) as well as with the peak in expression of VviNCED3 after veraison (Fig. 2e). RNAseq data confirmed the up-regulation of genes related to ABA biosynthesis [VIT_10s0003g03750 (VviNCED2); VIT_18s0001g10500 (VviCYP707A4); VIT_02s0087g00930 (VviNCED4, VviCCD4a); VIT_02s0087g00910 (VviNCED4, VviC$C D 4 b)$ ], ABA signaling [VIT_08s0058g00470 (VviPYL4); VIT_02s0012g01270 (VviPYRI)] and ABA catabolism [VIT_17s0000g02680 (VviBGLU44); VIT_06s0004g01430 (VviBGLU12)] after veraison, in BS berries (Fig. 2g). These expression profiles are in contradiction with ABA levels, which were gradually decreasing after veraison. However, strong increase of the main ABA storage metabolite ABA-GE indicated stimulation of ABA glucosylation (Fig. 2c), especially in BS berries. ABA-GE is a reversible glucoconjugate formed by UDP-glucosyltransferases as e.g. VviUGT73B4, which expression was strongly increased at veraison in healthy as well as BS berries (Fig. 2f). Nevertheless, the expression profile of VviUGT73B4 in BS berries cannot explain the enhanced levels of ABA-GE observed after veraison, stabilization of UGT73B4 protein might be responsible 
Table 1 Results of the quantification of phytohormone metabolites with HPLC-MS in healthy (H) and berry shrivel affected (BS) grape clusters collected 42, 55, 68, and 75 days after anthesis (DAA) in 2011

\begin{tabular}{|c|c|c|c|c|c|c|c|c|}
\hline \multirow{3}{*}{$\begin{array}{l}\text { Metabolite } \\
\text { (abbreviations) }\end{array}$} & \multicolumn{8}{|c|}{ Days after anthesis (mean value \pm standard error; data in $\mathrm{pmol} \mathrm{g}^{-1} \mathrm{DW}$ ) } \\
\hline & \multicolumn{2}{|l|}{42 (EL33) } & \multicolumn{2}{|l|}{55 (EL35) } & \multicolumn{2}{|l|}{$68(\mathrm{EL} 36 / 2)$} & \multicolumn{2}{|l|}{75 (EL37) } \\
\hline & Healthy & Berry shrivel & Healthy & Berry shrivel & Healthy & Berry shrivel & Healthy & Berry shrivel \\
\hline \multicolumn{9}{|l|}{ Auxins (AUX) } \\
\hline $\begin{array}{l}\text { Indole-3-actic } \\
\text { acid (IAA) }\end{array}$ & $44 \pm 10$ & $49 \pm 15$ & $39 \pm 13$ & $41 \pm 8$ & $32 \pm 15$ & $36 \pm 7$ & $25 \pm 2$ & $31 \pm 8$ \\
\hline $\begin{array}{l}\text { IAA-aspartate } \\
\text { (IAA-Asp) }\end{array}$ & $135 \pm 1 \mathrm{a}$ & $89 \pm 17 b$ & $134 \pm 11$ & $137 \pm 34$ & $874 \pm 238 \mathrm{a}$ & $232 \pm 27 b$ & $302 \pm 340$ & $228 \pm 42$ \\
\hline $\begin{array}{l}\text { IAA-gluta- } \\
\text { mate (IAA- } \\
\text { Glu) }\end{array}$ & $21 \pm 10$ & $9 \pm 4$ & $12 \pm 6$ & $11 \pm 6$ & $40 \pm 28$ & $19 \pm 6$ & $19 \pm 15$ & $19 \pm 7$ \\
\hline $\begin{array}{l}\text { oxo-IAA } \\
\text { (OxIAA) }\end{array}$ & $713 \pm 147 \mathrm{a}$ & $144 \pm 40 b$ & $176 \pm 54$ & $138 \pm 15$ & $159 \pm 38$ & $132 \pm 8$ & $99 \pm 83$ & $176 \pm 53$ \\
\hline $\begin{array}{r}\text { Phenylacetic } \\
\text { acid (PAA) }\end{array}$ & $2184 \pm 268$ & $1554 \pm 520$ & $2600 \pm 269$ & $2310 \pm 368$ & $1296 \pm 316$ & $2005 \pm 831$ & $1079 \pm 444$ & $1202 \pm 154$ \\
\hline $\begin{array}{l}\text { IAA-glucose } \\
\text { ester (IAA- } \\
\text { GE) }\end{array}$ & $6 \pm 3$ & $3 \pm 3$ & $2 \pm 2$ & $2 \pm 1$ & $1 \pm 1$ & $0 \pm 0$ & $1 \pm 1$ & $2 \pm 0$ \\
\hline $\begin{array}{l}\text { Indole- } \\
\text { 3-pyruvic } \\
\text { acid (IPyA) }\end{array}$ & $6 \pm 4$ & $5 \pm 2$ & $8 \pm 7$ & $4 \pm 4$ & $6 \pm 1$ & $7 \pm 4$ & $4 \pm 3$ & $10 \pm 2$ \\
\hline $\begin{array}{l}\text { Indole- } \\
\text { 3-acetamide } \\
\text { (IAM) }\end{array}$ & $4 \pm 3$ & $5 \pm 3$ & $4 \pm 3$ & $4 \pm 4$ & $4 \pm 4$ & $7 \pm 4$ & $1 \pm 1$ & $6 \pm 5$ \\
\hline $\begin{array}{l}\text { Indole-3-ace- } \\
\text { tonitrile } \\
\text { (IAN) }\end{array}$ & $5 \pm 3$ & $2 \pm 2$ & $4 \pm 3$ & $0 \pm 0$ & $0 \pm 0$ & $0 \pm 0$ & $0 \pm 0$ & $0 \pm 0$ \\
\hline \multicolumn{9}{|l|}{ Cytokinins (CK) } \\
\hline \multicolumn{9}{|c|}{ Active cytokinins } \\
\hline $\begin{array}{l}\text { Trans-zeatin } \\
(\mathrm{tZ})\end{array}$ & $18.8 \pm 5.2$ & $8.6 \pm 4.5$ & $4.6 \pm 1.0$ & $3.9 \pm 1.5$ & $19.4 \pm 5.8$ & $16.3 \pm 4.4$ & $21.9 \pm 3.9 \mathrm{a}$ & $11.9 \pm 2.3 b$ \\
\hline $\begin{array}{l}\text { Cis-zeatin } \\
\text { (cZ) }\end{array}$ & $1.6 \pm 2.1$ & $1.1 \pm 0.9$ & $1.3 \pm 0.6$ & $1.1 \pm 0.6$ & $0.7 \pm 0.6$ & $1.1 \pm 0.6$ & $6.9 \pm 2.8 \mathrm{a}$ & $0.4 \pm 0.2 b$ \\
\hline $\begin{array}{l}\text { Dihydrozea- } \\
\text { tin }(\mathrm{dZ})\end{array}$ & $19.8 \pm 6.8$ & $8.4 \pm 2.0$ & $6.7 \pm 3.6$ & $3.4 \pm 1.0$ & $24.4 \pm 9.0 \mathrm{a}$ & $3-4 \pm 0.6 b$ & $1.5 \pm 0.9$ & $4.4 \pm 1.6$ \\
\hline $\begin{array}{l}\text { Isopentenyl } \\
\text { adenin (iP) }\end{array}$ & $4.9 \pm 1.9$ & $1.7 \pm 1.0$ & $4.6 \pm 2.0$ & $2.8 \pm 0.4$ & $9.1 \pm 1.1 \mathrm{~b}$ & $13.8 \pm 1.5 \mathrm{a}$ & $108.0 \pm 5.9 \mathrm{a}$ & $5.8 \pm 1.1 \mathrm{~b}$ \\
\hline \multicolumn{9}{|c|}{ Cytokinin ribosides } \\
\hline $\begin{array}{l}\text { Trans-zeatin } \\
\text { riboside } \\
\text { (tZR) }\end{array}$ & $22.2 \pm 6.9$ & $7.7 \pm 1.2$ & $6.3 \pm 3.6$ & $11.8 \pm 1.1$ & $12.2 \pm 4.3$ & $2.6 \pm 0.7$ & $1.9 \pm 1.5$ & $4.3 \pm 3.3$ \\
\hline $\begin{array}{l}\text { Cis-zeatin } \\
\text { riboside } \\
(\mathrm{cZR})\end{array}$ & $1.3 \pm 0.4$ & $1.0 \pm 0.2$ & $1.0 \pm 0.5$ & $1.1 \pm 0.2$ & $0.8 \pm 1.0$ & $0.8 \pm 0.2$ & $0.3 \pm 0.1 b$ & $1.3 \pm 0.3 \mathrm{a}$ \\
\hline $\begin{array}{l}\text { Dihy- } \\
\text { drozeatin } \\
\text { riboside } \\
(\mathrm{dZR})\end{array}$ & $15.6 \pm 6.1$ & $8.7 \pm 2.7$ & $10.9 \pm 3.2$ & $8.0 \pm 1.0$ & $20.6 \pm 5.8 \mathrm{a}$ & $2.1 \pm 0.8 b$ & $1.4 \pm 1.2$ & $1.4 \pm 1.2$ \\
\hline $\begin{array}{l}\text { Isopenteny- } \\
\text { ladenosine } \\
\text { (iPR) }\end{array}$ & $1.4 \pm 1.3$ & $1.9 \pm 0.1$ & $0.7 \pm 0.5$ & $0.4 \pm 0.6$ & $0.1 \pm 0.3$ & $0.4 \pm 0.4$ & $0.1 \pm 0.2$ & $0 \pm 0$ \\
\hline
\end{tabular}


Table 1 (continued)

\begin{tabular}{|c|c|c|c|c|c|c|c|c|}
\hline \multirow{3}{*}{$\begin{array}{l}\text { Metabolite } \\
\text { (abbreviations) }\end{array}$} & \multicolumn{8}{|c|}{ Days after anthesis (mean value \pm standard error; data in $\mathrm{pmol} \mathrm{g}^{-1} \mathrm{DW}$ ) } \\
\hline & \multicolumn{2}{|l|}{42 (EL33) } & \multicolumn{2}{|l|}{55 (EL35) } & \multicolumn{2}{|l|}{$68(\mathrm{EL} 36 / 2)$} & \multicolumn{2}{|l|}{75 (EL37) } \\
\hline & Healthy & Berry shrivel & Healthy & Berry shrivel & Healthy & Berry shrivel & Healthy & Berry shrivel \\
\hline \multicolumn{9}{|c|}{ Deactivated cytokinins } \\
\hline $\begin{array}{l}\text { Trans-zeatin- } \\
\text { 7-glucoside } \\
\text { (tZ7G) }\end{array}$ & $11.2 \pm 1.6 \mathrm{a}$ & $6.0 \pm 0.9 \mathrm{~b}$ & $3.6 \pm 1.2$ & $3.8 \pm 1.7$ & $3.5 \pm 1.8$ & $2.2 \pm 0.7$ & $1.8 \pm 0.6$ & $3.7 \pm 2.1$ \\
\hline $\begin{array}{l}\text { Trans-zeatin- } \\
\text { 9-glucoside } \\
\text { (tZ9G) }\end{array}$ & $2.0 \pm 1.5$ & $0.9 \pm 0.5$ & $1.1 \pm 0.2$ & $0.6 \pm 0.3$ & $0.8 \pm 0.6$ & $0.4 \pm 0, .3$ & $0.3 \pm 0.4$ & $0.2 \pm 0.2$ \\
\hline $\begin{array}{l}\text { Dihydrozea- } \\
\text { tin-9-gluco- } \\
\text { side (dZ9G) }\end{array}$ & $11.3 \pm 3.3 \mathrm{a}$ & $1.4 \pm 0.5 \mathrm{~b}$ & $3.7 \pm 1.5$ & $4.1 \pm 1.8$ & $7.6 \pm 5.6$ & $2.0 \pm 0.3$ & $1.8 \pm 1.0$ & $3.7 \pm 2.8$ \\
\hline $\begin{array}{l}\text { Isopenteny- } \\
\text { ladenine- } \\
\text { 7-glucoside } \\
\text { (iP7G) }\end{array}$ & $6.3 \pm 3.5$ & $1.2 \pm 0.4$ & $2.9 \pm 1.4$ & $3.4 \pm 1.7$ & $1.6 \pm 0.7$ & $0.8 \pm 0.5$ & $0.6 \pm 0.4 \mathrm{~b}$ & $2.4 \pm 0.7 \mathrm{a}$ \\
\hline $\begin{array}{l}\text { Isopenteny- } \\
\text { ladenine- } \\
\text { 9-glucoside } \\
\text { (iP9G) }\end{array}$ & $0.1 \pm 0.1$ & $0.1 \pm 0.0$ & $0.1 \pm 0.1$ & $0.1 \pm 0.0$ & $0.2 \pm 0.2$ & $0.1 \pm 0.1$ & $0.0 \pm 0.0$ & $0.2 \pm 0.3$ \\
\hline \multicolumn{9}{|c|}{ Storage cytokinins } \\
\hline $\begin{array}{l}\text { Trans-zeatin- } \\
\text { O-glucoside } \\
\text { (tZOG) }\end{array}$ & $40.4 \pm 6.6 \mathrm{a}$ & $15.7 \pm 4.4 \mathrm{~b}$ & $16.3 \pm 7.6$ & $21.9 \pm 5.5$ & $19.3 \pm 9.2$ & $8.7 \pm 2.4$ & $11.2 \pm 3.7 \mathrm{~b}$ & $26.0 \pm 2.6 \mathrm{a}$ \\
\hline $\begin{array}{l}\text { Trans-zeatin } \\
\text { riboside- } \\
O \text {-glucoside } \\
\text { (tZROG) }\end{array}$ & $9.0 \pm 5.9$ & $2.5 \pm 1.8$ & $3.1 \pm 1.7$ & $3.7 \pm 3.0$ & $5.7 \pm 1.5$ & $5.1 \pm 1.0$ & $1.6 \pm 1.8$ & $4.5 \pm 2.3$ \\
\hline $\begin{array}{l}\text { Dihydrozeatin } \\
\text { riboside } \\
\text {-O-glu- } \\
\text { coside } \\
\text { (DRZOG) }\end{array}$ & $18.9 \pm 7.9 \mathrm{a}$ & $3.3 \pm 2.1 \mathrm{~b}$ & $11.2 \pm 5.7$ & $11.1 \pm 3.8$ & $43.3 \pm 13.6 \mathrm{a}$ & $1.9 \pm 2.1 \mathrm{~b}$ & $1.3 \pm 1.8$ & $1.6 \pm 1.8$ \\
\hline $\begin{array}{l}\text { Cis-zeatin- } \\
O \text {-glucoside } \\
\text { (cZOG) }\end{array}$ & $1.2 \pm 0.9$ & $0.5 \pm 0.3$ & $0.3 \pm 0.1$ & $0.3 \pm 0.2$ & $0.4 \pm 0.2$ & $0.3 \pm 0.3$ & $0.3 \pm 0.1$ & $0.4 \pm 0.4$ \\
\hline $\begin{array}{l}\text { Cis-zeatin } \\
\text { riboside } \\
\text {-O-glu- } \\
\text { coside } \\
\text { (cZROG) }\end{array}$ & $2.2 \pm 2.9$ & $1.5 \pm 1.6$ & $1.6 \pm 1.3$ & $2.0 \pm 0.3$ & $1.7 \pm 1.2$ & $2.4 \pm 3.5$ & $1.0 \pm 0.8$ & $1.5 \pm 1.6$ \\
\hline \multicolumn{9}{|c|}{ Cyotkinin precursors } \\
\hline $\begin{array}{l}\text { Trans-zeatin } \\
\text { riboside } \\
\text { monophos- } \\
\text { phate } \\
\text { (tZRMP) }\end{array}$ & $13.1 \pm 3.2 \mathrm{a}$ & $2.8 \pm 1.1 \mathrm{~b}$ & $4.2 \pm 4.2$ & $2.2 \pm 1.5$ & $2.8 \pm 1.2$ & $1.1 \pm 0.8$ & $1.9 \pm 1.4 \mathrm{~b}$ & $11.1 \pm 3.5 \mathrm{a}$ \\
\hline $\begin{array}{l}\text { Cis-zeatin } \\
\text { riboside } \\
\text { monophos- } \\
\text { phate } \\
\text { (cZRMP) }\end{array}$ & $1.5 \pm 1.6$ & $0.1 \pm 0.1$ & $0.2 \pm 0.2$ & $0.5 \pm 0.4$ & $0.8 \pm 0.5$ & $0.4 \pm 0.3$ & $0.3 \pm 0.2$ & $0.4 \pm 0.4$ \\
\hline
\end{tabular}


Table 1 (continued)

\begin{tabular}{|c|c|c|c|c|c|c|c|c|}
\hline \multirow{3}{*}{$\begin{array}{l}\text { Metabolite } \\
\text { (abbreviations) }\end{array}$} & \multicolumn{8}{|c|}{ Days after anthesis (mean value \pm standard error; data in $\mathrm{pmol} \mathrm{g}^{-1} \mathrm{DW}$ ) } \\
\hline & \multicolumn{2}{|l|}{42 (EL33) } & \multicolumn{2}{|l|}{$55(\mathrm{EL} 35)$} & \multicolumn{2}{|l|}{$68(\mathrm{EL} 36 / 2)$} & \multicolumn{2}{|l|}{75 (EL37) } \\
\hline & Healthy & Berry shrivel & Healthy & Berry shrivel & Healthy & Berry shrivel & Healthy & Berry shrivel \\
\hline $\begin{array}{l}\text { Dihydrozeatin } \\
\text { riboside } \\
\text { monophos- } \\
\text { phate } \\
\text { (DZRMP) }\end{array}$ & $13.7 \pm 3.2$ & $18.5 \pm 0.6$ & $7.0 \pm 2.4$ & $4.1 \pm 2.5$ & $12.9 \pm 7.3 \mathrm{a}$ & $0.5 \pm 0.5 b$ & $6.8 \pm 1.7$ & $2.6 \pm 2.8$ \\
\hline $\begin{array}{l}\text { Isopenteny- } \\
\text { ladenosine } \\
\text { monophos- } \\
\text { phate } \\
\text { (iPRMP) }\end{array}$ & $10.4 \pm 5.0$ & $5.1 \pm 3.5$ & $2.3 \pm 1.2$ & $2.1 \pm 0.8$ & $2.1 \pm 0.9$ & $4.1 \pm 3.8$ & $4.2 \pm 1.0 \mathrm{a}$ & $0.2 \pm 0.1 \mathrm{~b}$ \\
\hline \multicolumn{9}{|l|}{ Salicylic acid (SA) } \\
\hline $\begin{array}{l}\text { Salicylic acid } \\
\quad(\mathrm{SA})\end{array}$ & $855 \pm 114$ & $950 \pm 144$ & $625 \pm 26 b$ & $683 \pm 13 a$ & $423 \pm 124$ & $658 \pm 161$ & $258 \pm 9 b$ & $470 \pm 28 \mathrm{a}$ \\
\hline \multicolumn{9}{|l|}{ Jasmonic acid (JA) } \\
\hline $\begin{array}{l}\text { Jasmonic acid } \\
\text { (JA) }\end{array}$ & $169 \pm 62$ & $154 \pm 28$ & $74 \pm 16$ & $106 \pm 15$ & $32 \pm 12$ & $31 \pm 1$ & $16 \pm 4$ & $31 \pm 10$ \\
\hline $\begin{array}{l}\text { JA-isoleucine } \\
\text { (JA-Ile) }\end{array}$ & $15 \pm 5$ & $23 \pm 9$ & $28 \pm 8$ & $27 \pm 9$ & $7 \pm 3$ & $10 \pm 3$ & $4 \pm 2$ & $9 \pm 6$ \\
\hline cisOPDA & $144 \pm 64$ & $63 \pm 10$ & $634 \pm 11 \mathrm{a}$ & $163 \pm 24 b$ & $25 \pm 11$ & $22 \pm 4$ & $15 \pm 18$ & $17 \pm 9$ \\
\hline \multicolumn{9}{|l|}{ Ethylene (ET) } \\
\hline $\begin{array}{l}\text { 1-Aminocy- } \\
\text { clopropane- } \\
\text { 1-carboxylic } \\
\text { acid (ACC) }\end{array}$ & $9387 \pm 969 b$ & $29,535 \pm 3864$ a & $5499 \pm 1226 \mathrm{~b}$ & $10,108 \pm 1432 a$ & $7610 \pm 3045$ & $7455 \pm 2468$ & $6037 \pm 802 b$ & $\begin{array}{l}12,180 \pm \\
1693 \mathrm{a}\end{array}$ \\
\hline \multicolumn{9}{|c|}{ Absissic acid (ABA) } \\
\hline $\begin{array}{l}\text { Abscisic acid } \\
\text { (ABA) }\end{array}$ & $5232 \pm 1615$ & $3411 \pm 368$ & $23,698 \pm 5039$ & $16,670 \pm 4030$ & $15,125 \pm 3115$ & $20,515 \pm 2430$ & $7085 \pm 1247$ & $\begin{array}{l}10,215 \pm \\
1846\end{array}$ \\
\hline $\begin{array}{l}\text { ABA-glucose } \\
\text { ester (ABA- } \\
\text { GE) }\end{array}$ & $4552 \pm 265$ & $4828 \pm 252$ & $12,528 \pm 474 \mathrm{a}$ & $6732 \pm 622 b$ & $11,941 \pm 1672 b$ & $19,460 \pm 3475$ & $9332 \pm 1122 b$ & $\begin{array}{l}23,818 \pm \\
7126 a\end{array}$ \\
\hline $\begin{array}{l}\text { Phaseic acid } \\
\text { (PA) }\end{array}$ & $30 \pm 8$ & $44 \pm 10$ & $26 \pm 4$ & $26 \pm 2$ & $9 \pm 2$ & $10 \pm 12$ & $10 \pm 2$ & $4 \pm 4$ \\
\hline $\begin{array}{l}\text { Dihydrophaseic } \\
\text { acid (DPA) }\end{array}$ & $4730 \pm 1790$ & $7166 \pm 1385$ & $1106 \pm 268 \mathrm{a}$ & $694 \pm 83 b$ & $160 \pm 23$ & $202 \pm 77$ & $147 \pm 36 \mathrm{a}$ & $83 \pm 11 b$ \\
\hline \multicolumn{9}{|l|}{ Giberellins } \\
\hline $\begin{array}{l}\text { Giberellin } 4 \\
\left(\mathrm{GA}_{4}\right)\end{array}$ & $25 \pm 13$ & $11 \pm 5$ & $11 \pm 5$ & $8 \pm 3$ & $26 \pm 11$ & $15 \pm 23$ & $10 \pm 5$ & $6 \pm 3$ \\
\hline $\begin{array}{l}\text { Giberellin } 19 \\
\left(\mathrm{GA}_{19}\right)\end{array}$ & $51 \pm 10 a$ & $26 \pm 3 b$ & $27 \pm 6$ & $29 \pm 3$ & $12 \pm 4$ & $23 \pm 17$ & $21 \pm 2$ & $15 \pm 1$ \\
\hline
\end{tabular}

Data presented are mean values on dry weight basis \pm standard error $(n=3$ each). Statistical significant differences are indicated with different letters $(P<0.05)$

for this effect. In BS berries, ABA was quickly conjugated into ABA-GE and in parallel ABA-GE might be hydrolyzed by beta-D-glucopyranosyl abscisate beta-glucosidases [VviBGLU44 (VIT_17s0000g02680), VviBGLU12 (VIT_06s0004g01430)], as both genes were induced in BS berries. This might contribute to the high number of ABA responsive genes strongly expressed in $\mathrm{BS}$ berries during the ripening phase (Fig. $2 \mathrm{~g}$ ). The irreversible catabolism of ABA is mediated by 8'-hydroxylases (CYP707A1) to form phaseic acid (PA) and dihydrophaseic acid (DPA). In our study the contents of PA and DPA were relatively high in $\mathrm{H}$ and $\mathrm{BS}$ berries before veraison, decreased at veraison, being lowest during the ripening phase (Fig. 2b). The level of DPA at veraison was significantly lower in BS berries, which corresponded to a slightly reduced expression of ABA 8'-hydroxylase CYP707A1 (VIT_18s0001g10500) at EL35. 
(A)

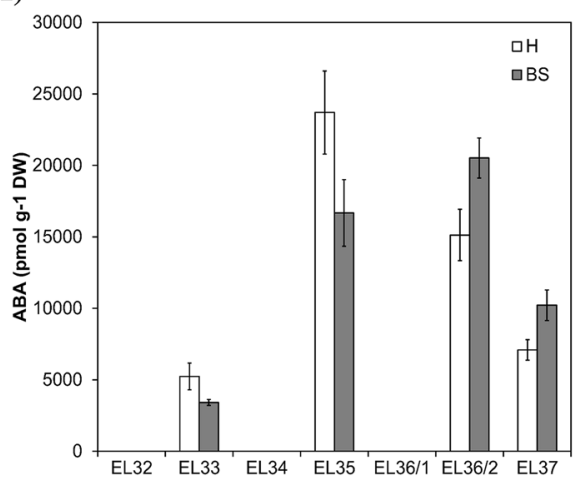

(B)

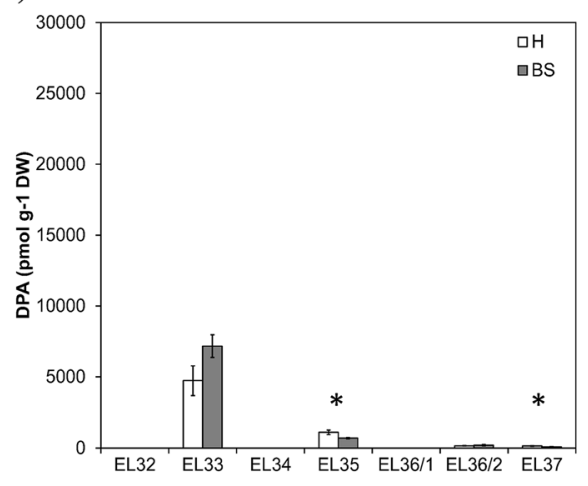

(C)

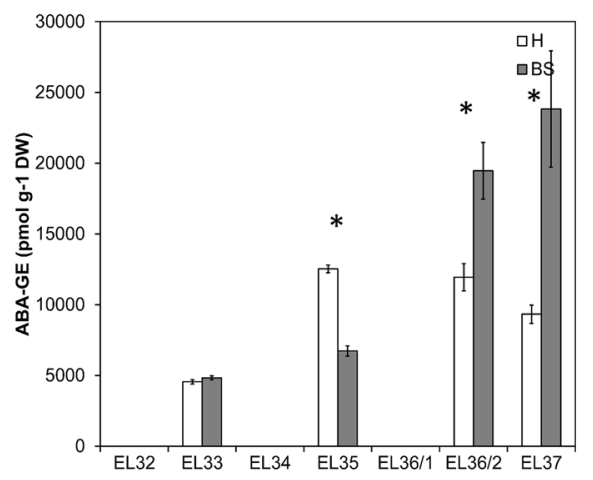

(D)

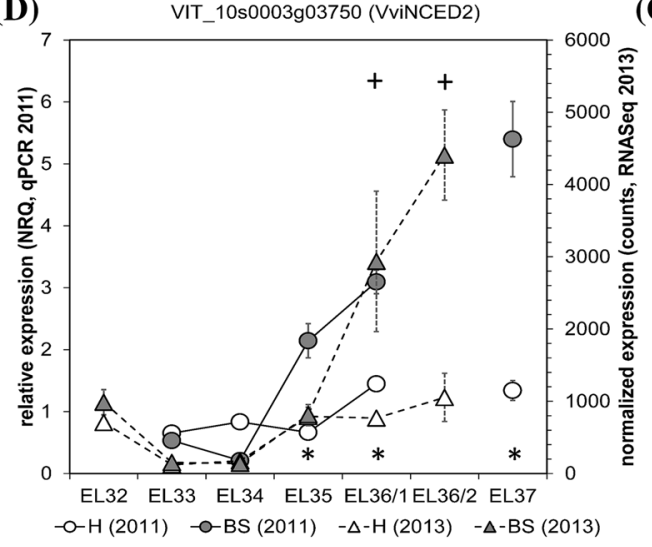

(E)

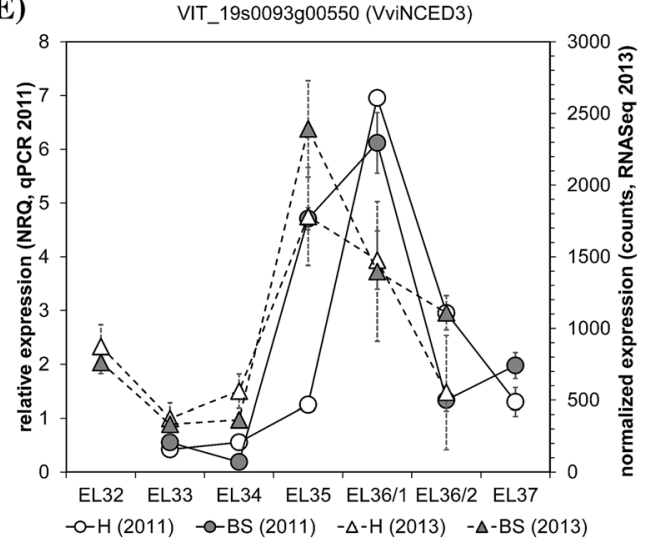

(F)

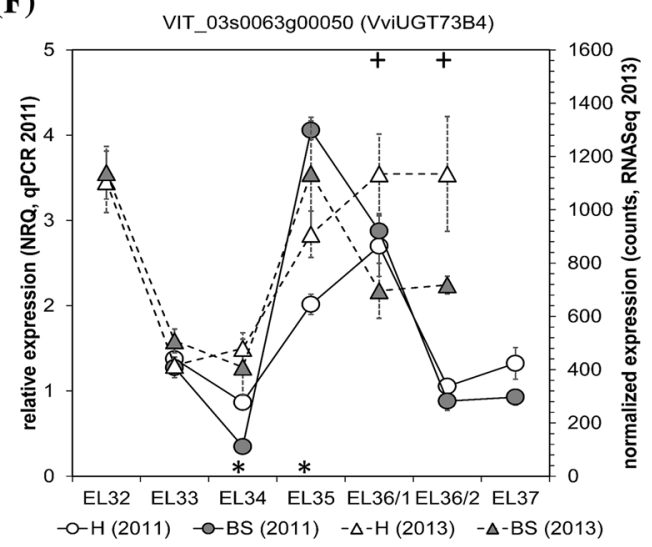

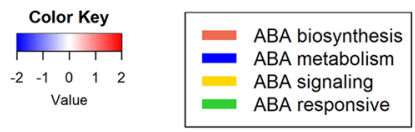

(G) EL: $: 32: 33: 34 ; 35 ; 36 ; 36$

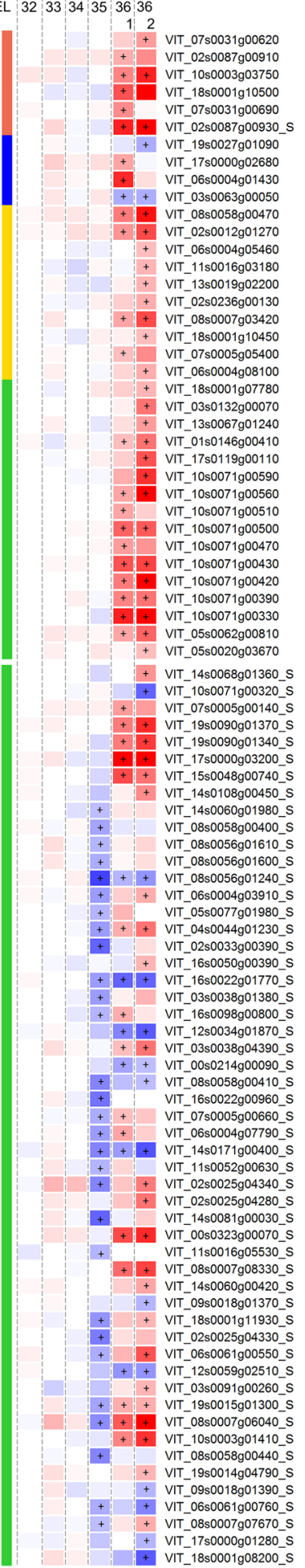

Fig. 2 Results obtained from analyses of ABA and its metabolites $(\mathbf{a}-\mathbf{c})$ and expression of ABA metabolism- and signal transductionrelated genes in healthy $(\mathrm{H})$ and berry shrivel (BS) grape clusters collected at six sampling dates (EL32, EL33, EL34, EL35, EL36/1, EL36/2, EL37) (d-g). a Abscisic acid (ABA) content, b phaseic acid content (DPA) and c ABA-glucose ester content (ABA-GE) in berry samples collected 2011. Expression of ABA biosynthetic genes genes VviNCED2 (d) and VviNCED3 (e) and ABA-metabolic gene Vvi-
UGT73B4 (f) determined by qPCR (samples 2011) in comparison with RNAseq analyses (samples 2013). g RNAseq results on genes related to ABA biosynthesis, metabolism, signaling, and responses in BS samples shown as $\operatorname{logFC}$. All data are mean values \pm standard error $(n=3$ each).Veraison (V) is defined as the start of coloring (EL35) and first BS symptoms were determined at EL36/1. Statistical significant differences are indicated with an asterisk (2011) and a plus symbol (2013) $(P<0.05)$ 
ABA signaling pathway is activated by ABA binding to receptors RCAR/PYR1/PYR1-LIKE (PYL) proteins and clade A protein phosphatases of type $2 \mathrm{C}$ (PP2C), which function as co-receptors. The complex formation results in PP2C degradation, releasing the associated protein kinases (SnRK2s) from PP2C inhibition (Ma et al. 2009; Nishimura et al. 2010). Among the genes differentially expressed in our study, ABA signaling genes at veraison were either not affected in BS berries or slightly repressed, whereas all of them were induced during the ripening phase (Fig. $2 \mathrm{~g}$ ), supporting the idea that $\mathrm{ABA}$ triggered gene expression is induced in $\mathrm{BS}$ berries after veraison. To conclude, $\mathrm{ABA}$ and ABA-GE along with the ABA signaling cascade are altered in BS-berries. We postulate that the reduced expression of the switch genes in BS berries at veraison (Savoi et al. 2019) is unlikely to be linked to ABA metabolites as a sole factor, in spite of the fact that several switch genes, which are modulated by ABA (Pilati et al. 2017) or belong to the ABA signal cascade (Savoi et al. 2019) were reduced in BS berries at veraison (Fig. 2g). The transcription profiles at EL36 and EL37 in BS berries resembled changes described for overripening berries (Cramer et al. 2014) and the post-harvest withering process (Zenoni et al. 2016), as recently discussed (Savoi et al. 2019). ABA has a central role in drought stress responses via the activation of ABA-dependent signaling pathways. It is interesting that several transcription factors are likewise activated by drought stress and BS (Savoi et al. 2017). Berry shrinking may be associated with stress responses via ABA function due to turgor loss. The elucidation of the differences in mechanism of drought stress responses, BS and post-harvest berry withering seems to be a prerequisite for understanding of BS symptoms during the berry ripening phase. The trigger for the induction of $\mathrm{ABA}$ biosynthesis genes at the transcriptional level as well as the physiological necessity to store high amounts of ABA-GE in vacuoles of BS berries remains unclear.
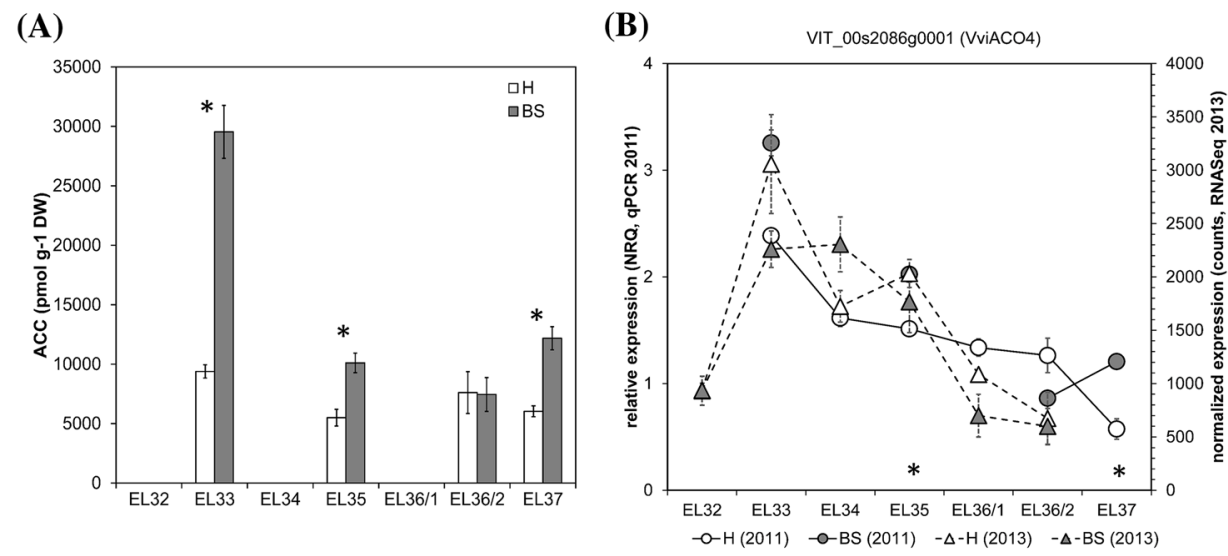

(D)

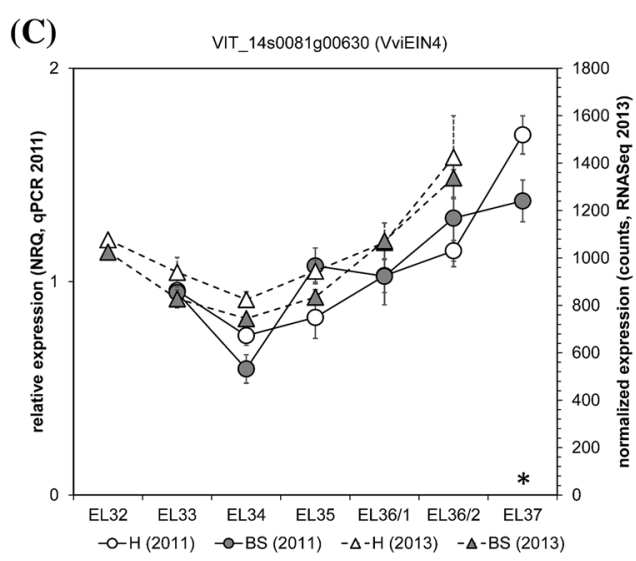

\begin{tabular}{l|l|l|l|l|l|l} 
EL & 32 & 33 & 34 & 35 & 36 & 36
\end{tabular}

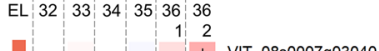
+ VIT_08s0007g03040 + + VIT_05s0020g00670 ++ VIT_11s0016g02380
+ VIT_05s0049g00410 ++ VIT_05s0049g00410 + + VIT_01s0011g05650
++ VIT $18 \mathrm{~s} 0001 \mathrm{~g} 07700$ ++ VIT_18s0001g07700 $+\quad$ VIT_06s0009g01380 $+\quad$ VIT_09s0002g01370 + VIT_08s0007g08580 VIT- $085007 \mathrm{~g} 08580$ + VIT $1150016 \mathrm{~g} 00660$ VIT_- $550029 \mathrm{~g} 00140$ VIT_07s0031900190 VIT_1850001g05890 VIT_16s0013g01050 VIT_16s0013g01110 VIT_09s0002g09140 VIT_06s0004g00490 VIT_16s0050g02400 VIT_06s0004g08190 VIT_05s0077g01860 VIT_ 0250234900130 VIT $12 \mathrm{~s} 0028 \mathrm{~g} 03270$ VIT 0350063900460 VIT 00 s $0179 \mathrm{~g} 00100$ VIT VIT 1300490160 (1) VIT 0550094900480 VIT_05s0094g00480 VIT_03s0091g00210 VIT_14s0060g01320 VIT_14s0060g01300 VIT_16s0100g00400_S VIT_05s0049g00510_S
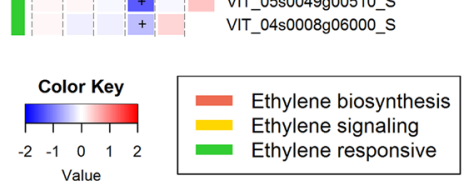

$-\mathrm{O}-\mathrm{H}(2011) \quad-\mathrm{O}-\mathrm{BS}(2011) \quad-\Delta-\mathrm{H}(2013) \quad-\Delta-\mathrm{BS}(2013)$

Fig. 3 Results obtained for ACC content (a) and the expression of ethylene metabolism- and signal transduction-related genes in healthy (H) and berry shrivel (BS) grape clusters collected at six sampling dates (EL32, EL33, EL34, EL35, EL36/1, EL36/2, EL37) (b-d). a ACC (ethylene precursor) content in berry samples collected 2011. Expression of ethylene biosynthetic gene VviACO4 (b) and signaling-related gene VviEIN4 (receptor) (c) determined by qPCR (sam- ples 2011) in comparison with RNAseq analyses (samples 2013). d RNAseq results on genes related to ethylene biosynthesis, metabolism, signaling, and responses in BS samples shown as logFC. All data are mean values \pm standard error ( $n=3$ each). Veraison (V) is defined as the start of coloring (EL35) and first BS symptoms were determined at EL36/1. Statistical significant differences are indicated with an asterisk (2011) and a plus symbol $(2013)(P<0.05)$ 


\section{Ethylene precursor ACC peaks in BS berries before veraison}

The ethylene precursor ACC was analyzed as a marker of ethylene production. ACC content in healthy berries did not differ among the four sampling time-points with no specific peak observed before or at the onset of ripening (Fig. 3a). Normally, in healthy berries ethylene peaks just before veraison 7-8 weeks after flowering with a duration of 1 (Muscat Hamburg) to 2 weeks (Cabernet Sauvignon) (Chervin et al. 2004; Sun et al. 2010). In our study, we possibly missed the ethylene peak in $\mathrm{H}$ berries as we did not analyze samples 1 week before veraison. Previous studies showed that the inhibition of ethylene accumulation had an effect on grape berry ripening and resulted in smaller berries, lower anthocyanin levels and higher acidity (Chervin et al. 2004), a symptomatology similar to BS. We determined an ACC peak in BS berries at EL33 about 14 days before veraison, which is 6 weeks after flowering and 1 week earlier as described in previous studies in healthy berries. The potential shift of the ACC peak in BS berries will be further studied to elucidate this cascade. ACC is a direct precursor of ethylene, its biosynthesis is the rate limiting step in ethylene formation. Additionally, ACC can be metabolized also to three conjugates (malonyl-ACC, $\gamma$-gametylACC, jasmonyl-ACC), with yet unknown biological roles and ACC is transported in plants to enable distant ethylene responses. Recently, a debate started whether ACC can have signaling function per se in plants (Van de Poel and Van Der Straeten 2014). The ACC peak in BS berries might have led to an ethylene signal, but other options like conjugation and transport cannot be excluded and should be considered in

Table 2 Results of an exogenous application of chemicals affecting the ethylene metabolism in grape berries with the aim to induce berry shrivel

\begin{tabular}{lllll}
\hline Treatment & Soluble solids $\left({ }^{\circ}\right.$ Brix $)$ & \multicolumn{3}{l}{ Grape clusters $($ each $N=30)$} \\
\cline { 3 - 5 } & & $\%$ Healthy & $\%$ BSN & $\%$ BS \\
\hline Control & $19.7( \pm 0.75)$ & 94 & 0 & 6 \\
Ethephon & $19.1( \pm 0.91)$ & $67 *$ & 33 & 0 \\
ACC & $19.0( \pm 0.97)$ & 80 & 3 & 17 \\
AVG & $19.8( \pm 0.89)$ & 94 & 0 & 6 \\
\hline
\end{tabular}

Applied treatments were: control; exogenous application of water (10 mL, 10 days before veraison), Ethephon $\left(1500 \mathrm{mg} \mathrm{L}^{-1} ; 2\right.$-chloroethylphosphonic acid, Bayer Crop Science), ACC [1000 $\mathrm{mg} \mathrm{L}^{-1}$; 1-aminocyclopropane-1-carboxylic acid, VBC-30426 Testproduct (10\%)] and AVG [250 $\mathrm{mg} \mathrm{L}^{-1}$; L- $\alpha$-(2-aminoethoxyvinyl)glycine hydrochloride, ReTain (15\%), Valent BioScience]. Effects of the treatments were evaluated visually by BS incidence and additionally by determining soluble solids ( ${ }^{\circ}$ Brix) before harvest (13.09.2016). Soluble solids are shown as mean values \pm standard deviation. Visual observation as percentage (\%) BS, BSN and H clusters

*Berries drop off very easily future approaches. The RNASeq and qPCR data (Fig. 3b-d) showed non-uniform expression profiles in BS berries and cannot explain the observed ACC peak at EL33 in BS berries. Before veraison, no genes related to ethylene metabolism were significantly differently expressed (Fig. 3d) and VviACO4 (Fig. 3b) was highly expressed at EL33 suggesting an active ethylene biosynthesis in both sample types. Several ethylene biosynthesis genes were suppressed (VIT_05s0020g00670, VviSAMT1; VIT_11s0016g02380, VviACO1) in BS berries after veraison, whereas other genes were induced (VIT_01s0011g0565, 1-aminocyclopropane1-carboxylate oxidase). Similar situation was found in case of ethylene responsive genes, whereas the majority of the genes involved in ethylene signaling were higher expressed in BS berries (Fig. 3d) and e.g. VviEIN3 (Fig. 3c). Recently, transcriptional biomarkers for the onset of ripening were identified in grapevine and individual ethylene response factors belonged to both negative as well as positive biomarkers (Fasoli et al. 2018), which may at least partially explain the observed undetermined expression profile in our study.

High ACC contents in BS berries led to the hypothesis that ethylene may trigger BS when applied before veraison. This hypothesis was tested by exogenous application of either ethephon (synthetic source of ethylene), ACC, AVG (aminoethoxyvinylglycine, inhibitor of ACC synthase, i.e. ethylene biosynthesis) or water and evaluation of BS symptoms of treated clusters. Treatment with ethephon led to a higher incidence of necrotic pedicels (similar to bunch stem necrosis) and berries dropped of the cluster very easily (Table 2). The application of ACC led to a slightly higher percentage of BS clusters (17\% as compared to $6 \%$ in water controls), whereas the application of AVG did not change BS incidence. The observed phenotype of necrotic pedicels after ethephon treatment differed from BS symptoms. Clusters of this treatment reduced the fruit detachment force and promoted the development of dry stem scar, which was also observed after ethephon application in order to produce high quality stemless fresh-cut table grapes (Ferrara et al. 2016). Similar results were observed using ACC treatments and a combined ACC and methyl jasmonate one, which strongly promoted the production of ethylene by grape berries and was in combination more effective to stimulate abscissionrelated processes (Uzquiza et al. 2014). In contrast, the ACC treatment in our study produced the BS phenotype. The timing, the applied concentrations and interaction with other phytohormones [e.g. with auxin for fruit abscission (Kuhn et al. 2014a)] may affect the physiological responses during berry growth and ripening, having different impacts on ethylene content. These pilot data call for further tests to strengthen this conclusion and provide functional proof. 
(A) VIT_01s0010g01200 (VviDWARF1)

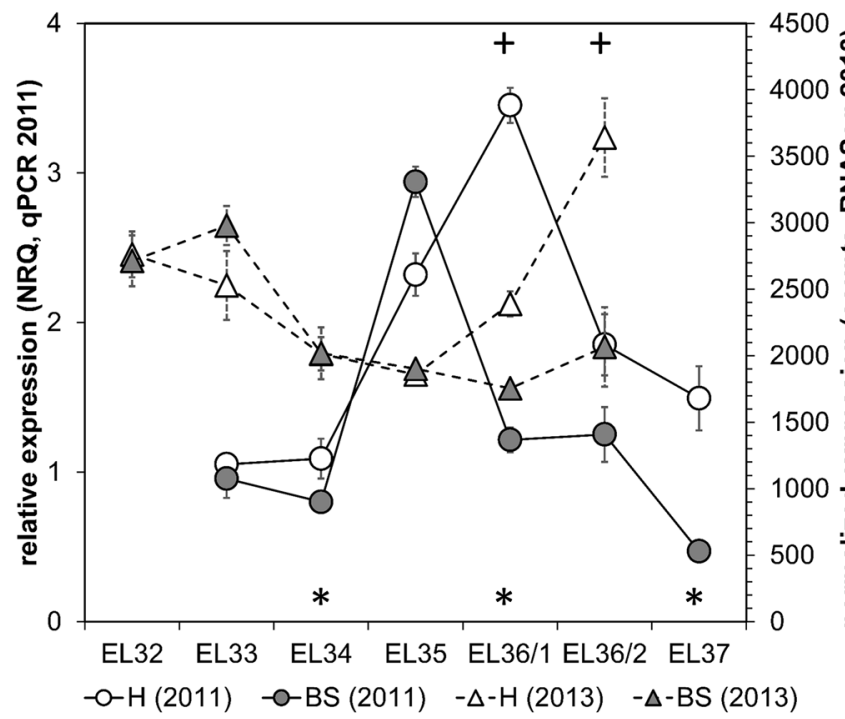

(B) VIT_14s0083g01110 (VviBR6OX1)

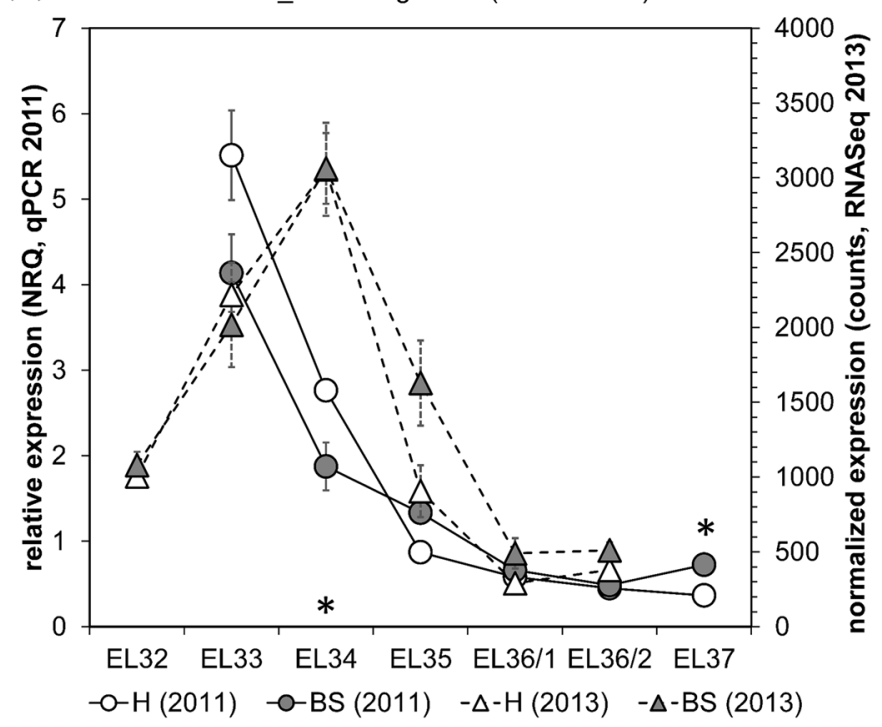

(C)
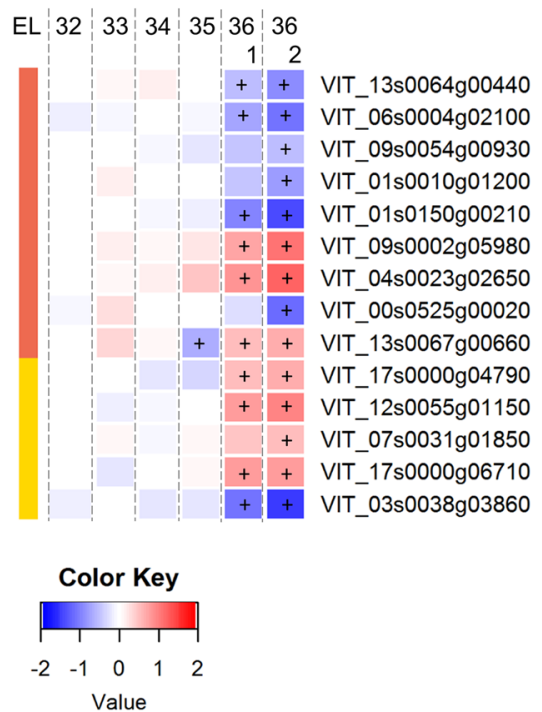

Brassinosteroid biosynthesis Brassinosteroid signaling
Fig. 4 Brassinosteroid-related (BR) gene expression in healthy $(\mathrm{H})$ and berry shrivel (BS) grape clusters collected at six sampling dates (EL32, EL33, EL34, EL35, EL36/1, EL36/2, EL37) (a-c). Expression of BR biosynthetic genes: a VviDWARF1 and b VviBR6OX1 determined by qPCR (samples 2011) in comparison with RNAseq analyses (samples 2013). c RNAseq results on genes related to BR

\section{Brassinosteroid biosynthesis was suppressed in ripening $B S$ berries}

Brassinosteroids (BR) are a class of phytohormones with a diverse set of functions, e.g. promotion of cell expansion and cell elongation (together with auxin), stimulation of vascular differentiation, pollen tube formation and skotomorphogenesis as recently reviewed (Baghel et al. 2019). In grape berries BRs are increased at the onset of biosynthesis and BR signaling in BS samples. All data are mean values \pm standard error $(n=3$ each). Veraison $(V)$ is defined as the start of coloring (EL35) and first BS symptoms were determined at EL36/1. Statistical significant differences are indicated with an asterisk (2011) and a plus symbol $(2013)(P<0.05)$

grape berry ripening and their exogenous applications can induce ripening (Symons et al. 2006). Therefore they are considered as early signals for ripening, possibly through ethylene content modulation (Ziliotto et al. 2012). Our gene expression analyses confirmed the induction of VviBR6OX1 (brassinosteroid-6-oxidase) before veraison in both years in healthy grape berries (Fig. 4b). The values in BS berries were very similar, suggesting that BR biosynthesis is not affected before veraison which 
is in contrast to the situation observed after veraison. The expression of another BR biosynthesis gene $V v i D$ WARF 1 ( $22 \alpha$-hydroxylase) was higher after veraison in healthy berries as compared with BS berries, which also exhibited a shift in expression peak towards veraison in 2011 (Fig. 4a). RNAseq data showed down-regulation of the expression of several BR biosynthesis genes in BS berries (Fig. 4d). The role of BRs during the grape berry
(A)

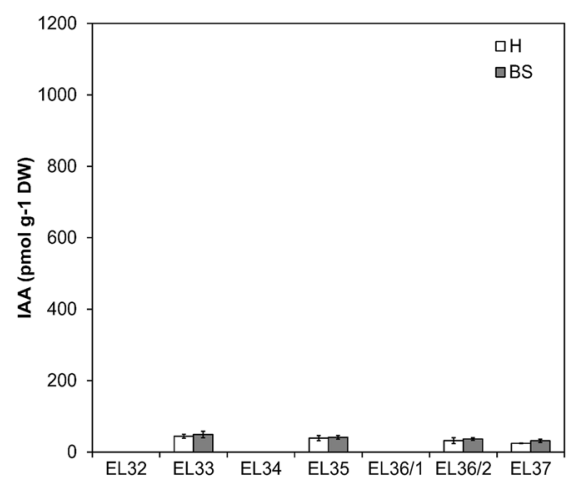

(B)

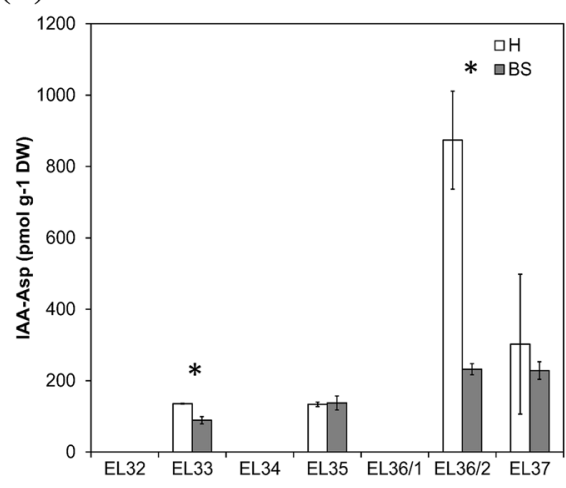

(C)

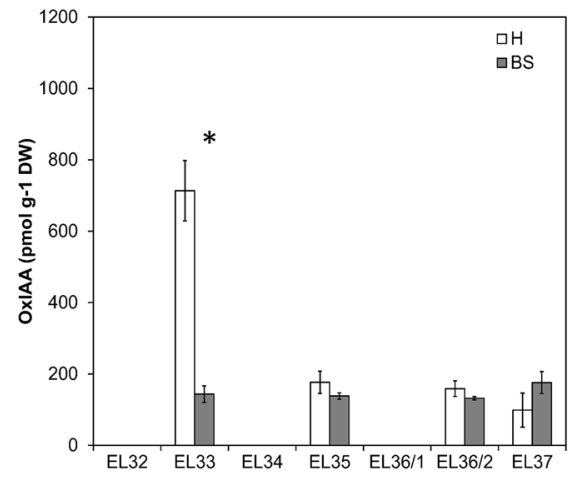

Fig. 5 Results obtained from analyses of IAA and its metabolites (a-c) and expression of IAA metabolism- and signal transductionrelated genes in healthy $(\mathrm{H})$ and berry shrivel (BS) grape clusters collected at six sampling dates (EL32, EL33, EL34, EL35, EL36/1, EL36/2, EL37) (d-f). a Indole-3-acetic acid (IAA) content, b IAAaspartate (IAA-Asp) content and c oxIAA content in berry samples collected 2011. Expression of IAA biosynthetic gene VviYUC10 (d) and IAA metabolism-related gene $V v i G H 3-2$ (e) determined by qPCR

(E)
(F)
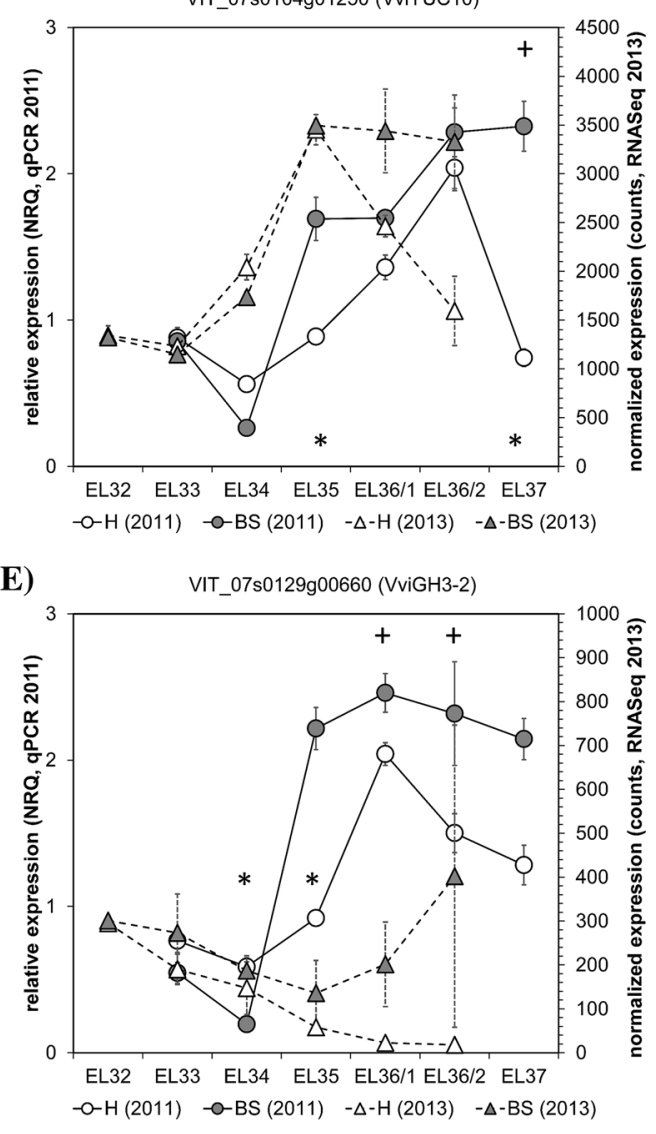

\begin{tabular}{l|l|l|l|l|l|l|l|l|l|l}
\hline EL & 32 & 33 & 34 & 35 & 36 & 36 \\
& & & & & 1 & 2 \\
\hline
\end{tabular} VIT_00s0394g00040 VIT_18s0001g15100 VIT_07s0104g01260 VIT_07s0104g01250 VIT_08s0007g02740 VIT_01s0026g00820 VIT_11s0016g02700 VIT_03s0091g00310 VIT_07s0129g00660 VIT_19s0014g04690 VIT $0750104 \mathrm{~g} 00800$ VIT $07 \mathrm{~s} 0104 \mathrm{~g} 01230$ VIT 08s0040g01810 + VIT_08s0040g01810
+ VIT_10s0003g04100 VIT_10s0003g04100
VIT_15s0046g00290 VIT_18s0001g13930 VIT_18s0001g08090 + VIT_09s0002g05160 VIT 0950002004080 VIT 0750141900290 VIT $1250035 \mathrm{~g} 01840$ VIT_- $1250035 \mathrm{~g} 01840$ VIT_19s0090g01650 VIT_13s0067g00330 + VIT_07s0031g02200

++ VIT_04s0044g01870 VIT_04s0023g00320 VIT_17s0000g02420 VIT 03 s0038g02140 VIT_04s0023g00530 VIT_03s0038g01180 VIT_18s0086g00410 VIT_18s0001g06390 + VIT_13s0064g01510 VIT_11s0016901190 VIT_08s0007g01650 VIT 0150011905000 VIT VIT_00s0313g00030 VIT_09s0002g00650 VIT_14s0030g02310 VIT_07s0141g00270 VIT_05s0020g04680 VIT_14s0030g01980 VIT_06s0061g00700 VIT_06s0004g04150 VIT_10s0405g00040 VIT_05s0062g00850 VIT_12s0057g00420 VIT_11s0016g03540 VIT_15s0048g00530 VIT_07s0031g02740 VIT_15s0048g02860 + VIT_04s0023g03230 + VIT_03s0038g00950 + VIT 1450060901720 VIT 095000200700 VIT_10s0003g00090 VIT $0450008 \mathrm{~g} 02800$ VIT 1900014023130 VIT_19s0014g03130 + VIT_10s0597g00010 + VIT_05s0020g04160 VIT_16s0098g01150_S (samples 2011) in comparison with RNAseq analyses (samples 2013) (g). RNAseq results on genes related to IAA biosynthesis, signaling, transport, and responses in BS samples shown as $\operatorname{logFC}$. All data are mean values \pm standard error ( $n=3$ each). Veraison $(\mathrm{V})$ is defined as the start of coloring (EL35) and first BS symptoms were determined at EL36/1. Statistical significant differences are indicated with an asterisk (2011) and a plus symbol $(2013)(P<0.05)$ 
ripening process was recently analyzed in more detail by applying exogenous BR (24-epibrassinolide) and the BR synthesis inhibitor brassinazole at the onset of veraison (Xu et al. 2015). Thereby exogenous BR application induced the activity as well as the expression of invertases and mono- and disaccharide transporters, while contrasting effects were obtained with brassinazole. Higher brassinolide contents led to a negative feedback to BR biosynthesis, resulting in down-regulation of the expression of VviBR6OX1 and VviDWARF1, while the BR receptor VviBRIl was induced (Xu et al. 2015). BR biosynthesis seems to be reduced in BS berries, but currently we can only speculate about metabolite contents and their consequences for sugar accumulation. Previous studies reported an increased expression of invertases, hexose transporters, sucrose transporters and sucrose synthase in BS berries during the ripening phase as compared to controls, while tonoplast monosaccharide transporters, glycolysis and TCA cycle were suppressed (Savoi et al. 2019). Reduced BR biosynthesis could be the result of a negative BR feedback inducing enzymes and transporters for sugar accumulation. However without BR analyses the link remains speculation. Strikingly, BR biosynthesis is suppressed in BS berries, while many other phytohormone pathways are induced during the ripening phase.

\section{Inconsistent auxin responses}

Auxins play an important role during the first growing phase of grape berries, but act as inhibitors of the ripening process in both climacteric and non-climacteric fruits. Low auxin levels are required at the onset of grape ripening, while conjugated forms of IAA usually increase with veraison (Bottcher et al. 2013b; Kumar et al. 2014). The application of NAA (1-napthalene acetic acid) to pre-veraison grapes delayed the onset of growth resumption, the accumulation of sugars and anthocyanins, the decrease in organic acid content and the increase in ABA levels (Bottcher et al. 2011, 2012). It seems that the entire berry ripening program is put on hold by auxin, which also delayed anthocyanin biosynthesis and switch gene expression in BS berries (Griesser et al. 2018; Savoi et al. 2019). Our data showed stable and not significantly different contents of IAA in $\mathrm{H}$ and BS samples (Fig. 5a). In healthy berries we observed a peak of 2-oxindole-3-acetic acid (oxIAA) at EL33 which is missing in BS berries (Fig. 5c). The complex interaction between auxin and ethylene was described (Muday et al. 2012), taking place also in pre-veraison grape berries (Bottcher et al. 2013b). Pre-ripening application of the ethylene-releasing compound Ethrel delayed the onset of ripening and enhanced the expression of genes for auxin biosynthesis and the amounts of IAA and IAA-Asp in grapes (Bottcher et al. 2013b). Other studies support the proposed cross talk between ethylene and auxin signaling in fruit ripening, although not all biochemical mechanism are known (Shin et al. 2016; Tadiello et al. 2016). Ethylene regulates root growth via effects on auxin biosynthesis, transport and signaling and ethylene as well as auxin regulate the transcription of key genes associated with the biosynthesis of both hormones (Fernie and Alseekh 2018; Muday et al. 2012). Whether the ACC peak in BS berries at EL33 actually influenced the auxin metabolism (e.g. oxIAA levels and induced auxin biosynthesis) needs to be determined as well as if auxin could influence ACC synthase at an early stage of berry growth. Experimental approaches are being conducted to decipher their interaction in grape berry ripening and maybe also in the sense of BS induction.

Auxin homeostasis in cells is tightly controlled and free IAA content is modulated by conjugation and/or oxidation (Ljung 2013). IAA can either be conjugated via ester linkages to glucose by UDP-glucosyl transferases or to amino acids by the Gretchen hagen3 (GH3) family of IAA-amido synthetases, or oxidized via IAA oxidase to form oxIAA, which seems to be the major catabolic pathway (Zhang and Peer 2017). Low auxin levels are necessary to start ripening as indicated by down-regulation of several auxin related and response genes at the onset of ripening (Bottcher et al. 2011; Fasoli et al. 2018). Only two genes, namely VviIAA19 and VviSAUR29, were identified as positive biomarkers for the onset of ripening (Fasoli et al. 2018; Palumbo et al. 2014). Both genes, VviSAUR29 (VIT_15s0048g02860) and VviIAA19 (VIT_18s0001g08090), were only slightly induced at EL36/2 in BS berries and no difference in expression was observed at veraison. The expression analyses in BS berries provided rather a non-conclusive picture, as several auxin related genes reacted in opposite ways. The enhancing trend in the auxin biosynthesis could be observed in BS berries after veraison (Fig. 5f), while an increased levels of IAAaspartate (IAA-Asp) were determined in $\mathrm{H}$ but not in BS berries after veraison, although an induction of $\mathrm{VviGH3}-2$ (VIT_07s0129g00660) (Fig. 5e), one of the genes responsible for the IAA conjugation, was detected. The expression pattern of VviGH3-2 differed between the tested years (Fig. 5e). Recently a cultivar specificity of the expression profile of this gene was described (Coelho et al. 2019), which supports the pattern observed in 2013 in our study.

\section{Major decrease of the cytokinin iP in BS berries during the ripening phase}

The crucial cytokinin role is stimulation of cell division and differentiation, e.g. in shoot and flower development, female gametophyte development as well as vascular tissue and root meristem development and root nodule formation (Wybouw and De Rybel 2019). In plants, the active cytokinins are trans-zeatin (tZ), N6-( $\Delta^{2}$-isopentenyl)-adenine (iP), cis-zeatin (cZ), and dihydrozeatin (Haberer and Kieber 
(A)

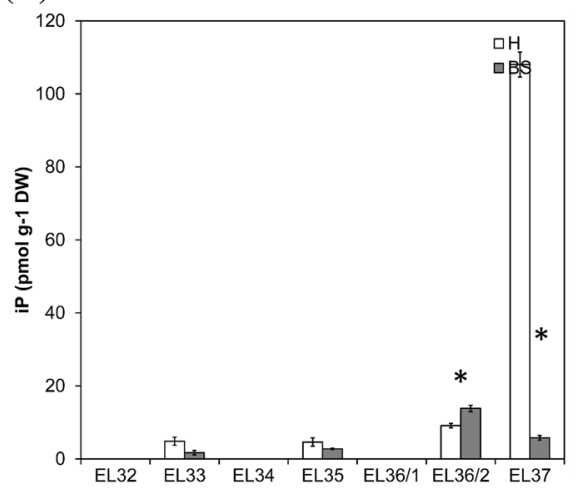

(C)

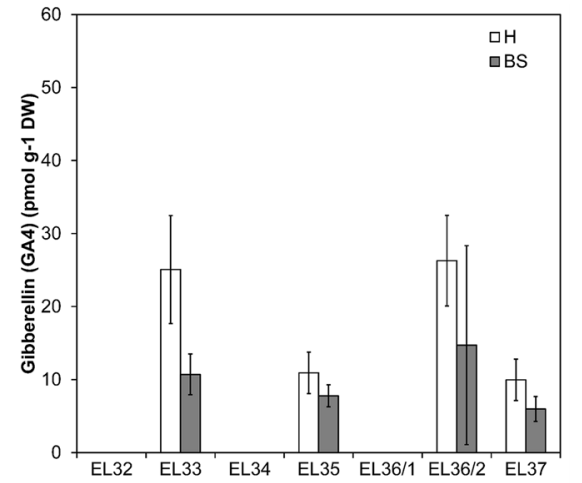

(B)

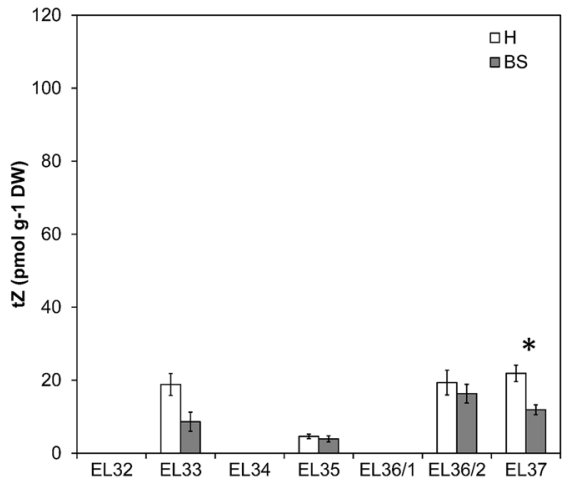

(D)

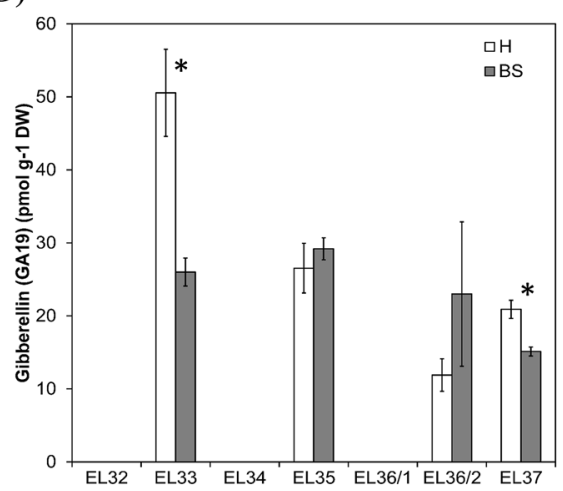

(E)

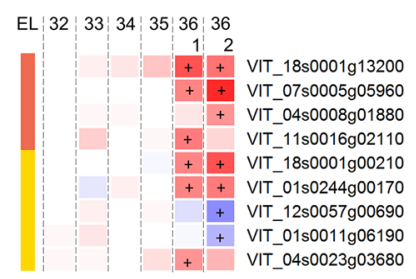

(F)

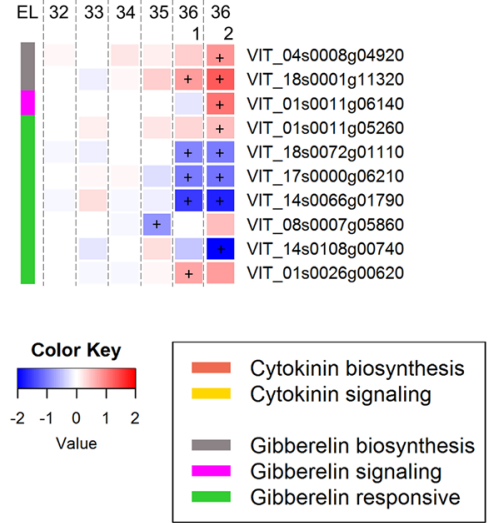

Fig. 6 Results obtained from metabolite analyses of cytokinins and gibberellins (a-d) and expression of cytokinin and gibberellin metabolism- and signal transduction-related genes in healthy $(\mathrm{H})$ and berry shrivel (BS) grape clusters collected at six sampling dates (EL32, EL33, EL34, EL35, EL36/1, EL36/2, EL37) (e-f). a Isopentenyladenine (iP) content, $\mathbf{b}$ trans-zeatin (tZ) content, $\mathbf{c} \mathrm{GA}_{4}$ (active gibberellin) content and $\mathbf{d ~ G A _ { 1 9 }}$ (gibberellin precursor) content in berry samples collected 2011. e RNAseq results on genes related to cytokinin

2002). Cytokinins peak after fertilization and during early fruit growth, stimulating cell division (Gillaspy et al. 1993). Their roles at later stages of fruit ripening are less described. Synchronous increase of iP together with soluble solids was observed in ripening berries supporting the important function of iP in the establishment and maintenance of sink strength (Bottcher et al. 2013a, 2015). In our study in total 22 cytokinin metabolites were analyzed (Table 1 ). We focused on $\mathrm{iP}$ and $\mathrm{tZ}$ as the most abundant active cytokinins in grapes. The level of $\mathrm{tZ}$ was transiently reduced around veraison (EL35) (Fig. 6b) which is in accordance with a previous report (Bottcher et al. 2015). The content of iP strongly increased in $\mathrm{H}$ berries at EL37 but not in BS berries (Fig. 6a). It has been proposed that the role of iP in ripening berries is related to the expansion driven growth after veraison and the high rate of sugar accumulation to establish and maintain sink strength, as shown for vegetative organs (Bottcher et al. 2015; Kuiper 1993). BS berries fail to accumulate sugars in high concentrations after veraison, although sugar biosynthesis and signaling and $\mathbf{f}$ RNAseq results on genes related gibberellin biosynthesis, signaling, and responses in BS samples shown as $\log$ FC. All data are mean values \pm standard error $(n=3$ each). Veraison $(\mathrm{V})$ is defined as the start of coloring (EL35) and first BS symptoms were determined at EL36/1. Statistical significant differences are indicated with an asterisk (2011) and a plus symbol (2013) $(P<0.05)$

accumulation starts, being stopped thereafter between 10 and $14^{\circ}$ Brix (Griesser et al. 2012, 2018). Lower sink strength or loss of sink strength could be a possible explanation. The observed failed accumulation of iP in ripening BS berries could be one piece of information to support this conclusion. Nevertheless further studies are needed to investigate both the role of cytokinins, and especially of iP, in grape berries during the ripening phase in general and their role in BS symptom development related with sugar accumulation.

Gibberellins have a role in cell division and enlargement and higher contents of active gibberellins have so far been found in flowers and young developing fruits, whereas significant levels were not detected from veraison onwards (Bottcher et al. 2013a; Symons et al. 2006). GA 4 and $\mathrm{GA}_{19}$ decreased during ripening and their lower contents were determined in BS berries, which correlated well with lower expression of gibberellin responsive genes in BS berries (Fig. 6c, d, f). No information on gibberellins during later 
grapevine ripening stages is available and the consequences for BS berries after veraison are unclear.

\section{Conclusion}

The aim of this study is to characterize the processes that occur in BS phenotypes during the berry growth and ripening phases. Main symptoms of BS phenotypes are decreased sugar contents due to delayed sugar accumulation and weak sink strength while both rachis and pedicels remain green in color. In previous studies we showed that anthocyanin biosynthesis is delayed in ripening leading to lower anthocyanin contents (Griesser et al. 2018; Savoi et al. 2019) and the expression of 67 switch genes is reduced at veraison in BS berries (Savoi et al. 2019).

Here we show that many phytohormonal biosynthesis pathways (ABA, auxin, and cytokinin) are induced in BS berries after veraison on the transcriptional level, while ethylene and brassinosteroids are suppressed. One may question which process(es) keeps BS berries metabolically active during the ripening phase, as shedding or abscission of such berries would be resourceful. We describe two distinct phytohormone profiles in BS berry phenotypes: pre- and postveraison. Firstly, an ACC peak about 2 weeks before veraison was determined in BS berries and the reciprocal ethyleneauxin crosstalk needs to be taken into consideration in a next step. The application of ACC pre-veraison led to BS symptoms while ethephon induced berry abscission. Temporal and spatial sensitivity towards phytohormone changes in grape berries throughout the ripening process and its consequences both in healthy and induced BS phenotypes are unclear. Secondly, we propose that the induction of several phytohormone pathways prevent fruit abscission as e.g. observed with bunch stem necrosis or sunburn, post-veraison. The similarities and differences in transcriptional patterns of ripening disorders and withering processes need to be determined as well as the role of iP (and possibly also of ABA-GE and IAA-Asp) in berry ripening as well as the consequences of its decreased accumulation for sink activity in berries. Sophisticated approaches and defined experiments are needed to decipher in detail the pivotal role of phytohormones in BS induction pre-veraison and in the development of BS symptoms after veraison. The presented study and previously published data provide valuable knowledge for these next steps.

Acknowledgements Open access funding provided by Austrian Science Fund (FWF). Parts of the study were funded by ASEA-UNINET Ernst Mach Grant. RNASeq study was performed in the frame of the project P28966-B29 funded by the Austrian Science Fund (FWF). We also thank Martina Kolarek a master student supporting the project.
Author contribution MG conceived and designed the study, coordinated the sampling strategy, decided on analyses conducted, supervised qPCR and RNASeq analyses, performed data analyses, interpretation of results and drafted the manuscript; SS carried out RNA extraction from samples 2013 and performed RNASeq analyses; PS extracted RNA from samples 2011 and performed qPCR; RV supervised metabolite analyses and critically revised the manuscript; PD performed phytohormone analysis by LC/MS; AF supervised the study and all the experiments and critically discussed and revised the manuscript. All authors read and approved the final manuscript.

Availability of data and materials All raw transcriptomics reads have been deposited in NCBI Sequence Read Archive in the BioProject PRJNA436693 with SRA accession SRP134067 https://www.ncbi.nlm. nih.gov/bioproject/PRJNA436693/

\section{Compliance with ethical standards}

Conflict of interest The authors declare that they have no competing interests.

Open Access This article is licensed under a Creative Commons Attribution 4.0 International License, which permits use, sharing, adaptation, distribution and reproduction in any medium or format, as long as you give appropriate credit to the original author(s) and the source, provide a link to the Creative Commons licence, and indicate if changes were made. The images or other third party material in this article are included in the article's Creative Commons licence, unless indicated otherwise in a credit line to the material. If material is not included in the article's Creative Commons licence and your intended use is not permitted by statutory regulation or exceeds the permitted use, you will need to obtain permission directly from the copyright holder. To view a copy of this licence, visit http://creativecommons.org/licenses/by/4.0/.

\section{References}

Baghel M, Nagaraja A, Srivastav M, Meena NK, Kumar MS, Kumar A, Sharma RR (2019) Pleiotropic influences of brassinosteroids on fruit crops: a review. Plant Growth Regul 87:375-388

Bondada B (2016) Nutritonal aspects of grapes (Vitis vinifera L.) clusters afflicted with SOUR shrivel is related to functionality of its vascular tissues. Am J Plant Sci 7:194-200

Bondada B, Keller M (2012a) Morphoanatomical symptomatology and osmotic behavior of grape berry shrivel. J Am Soc Hortic Sci 137:20-30

Bondada BR, Keller M (2012b) Not all Shrivels are created equalmorpho-anatomical and compositional characteristics differ amon different shrivel types that develop during ripening of grape (Vitis vinifera L.) berries. Am J Plant Sci 3:879-898

Bottcher C, Keyzers RA, Boss PK, Davies C (2010) Sequestration of auxin by the indole-3-acetic acid-amido synthetase GH3-1 in grape berry (Vitis vinifera L.) and the proposed role of auxin conjugation during ripening. J Exp Bot 61:3615-3625. https://doi. org/10.1093/jxb/erq174

Bottcher C, Harvey K, Forde CG, Boss PK, Davies C (2011) Auxin treatment of pre-veraison grape (Vitis vinifera L.) berries both delays ripening and increases the synchronicity of sugar accumulation. Aust J Grape Wine Res 17:1-8

Bottcher C, Boss PK, Davies C (2012) Delaying Riesling grape berry ripening with a synthetic auxin affects malic acid metabolism and sugar accumulation, and alters wine sensory characters. Funct Plant Biol 39:745-753. https://doi.org/10.1071/Fp12132 
Bottcher C, Boss PK, Davies C (2013a) Increase in cytokinin levels during ripening in developing Vitis vinifera cv. shiraz berries. Am J Enol Viticult 64:527-531

Bottcher C, Burbidge CA, Boss PK, Davies C (2013b) Interactions between ethylene and auxin are crucial to the control of grape (Vitis vinifera L.) berry ripening. BMC Plant Biol 13(1):222

Bottcher C, Burbidge CA, Boss PK, Davies C (2015) Changes in transcription of cytokinin metabolism and signalling genes in grape (Vitis vinifera $\mathrm{L}$.) berries are associated with the ripening-related increase in isopentenyladenine. BMC Plant Biol 15(1):223

Castellarin SD et al (2016) Characterization of major ripening events during softening in grape: turgor, sugar accumulation, abscisic acid metabolism, colour development, and their relationship with growth. J Exp Bot 67:709-722

Chervin C, El-Kereamy A, Roustan JP, Latche A, Lamon J, Bouzayen M (2004) Ethylene seems required for the berry development and ripening in grape, a non-climacteric fruit. Plant Sci 167:1301-1305

Chervin C, Terrier N, Ageorges A, Ribes F, Kuapunyakoon T (2006) Influence of ethylene on sucrose accumulation in grape berry. Am J Enol Viticult 57:511-513

Coelho J et al (2019) The study of hormonal metabolism of Trincadeira and Syrah cultivars indicates new roles of salicylic acid, jasmonates, ABA and IAA during grape ripening and upon infection with Botrytis cinerea. Plant Sci 283:266-277

Coombe BG, Hale CR (1973) The hormone content of ripening grape berries and the effects of growth substance treatments. Plant Physiol 51:629-634

Cramer GR et al (2014) Transcriptomic analysis of the late stages of grapevine (Vitis vinifera cv. Cabernet Sauvignon) berry ripening reveals significant induction of ethylene signaling and flavor pathways in the skin. BMC Plant Biol 14(1):370

Crespo-Martinez S, Sobczak M, Rozanska E, Forneck A, Griesser M (2019) The role of the secondary phloem during the development of the grapevine berry shrivel ripening disorder. Micron 116:36-45. https://doi.org/10.1016/j.micron.2018.09.012

Dobrev PI, Kaminek M (2002) Fast and efficient separation of cytokinins from auxin and abscisic acid and their purification using mixed-mode solid-phase extraction. J Chromatogr A 950:21-29

Dobrev PI, Vankova R (2012) Quantification of abscisic acid, cytokinin, and auxin content in salt-stressed plant tissues. Methods Mol Biol 913:251-261. https://doi.org/10.1007/978-1-61779 -986-0_17

Fasoli M et al (2018) Timing and order of the molecular events marking the onset of berry ripening in grapevine. Plant Physiol 178:11871206. https://doi.org/10.1104/pp.18.00559

Fernie AR, Alseekh S (2018) Defining the convergence of ethylene and auxin signaling in tomato. New Phytol 219:479-481

Ferrara G, Mazzeo A, Matarrese AMS, Pacucci C, Trani A, Fidelibus MW, Gambacorta G (2016) Ethephon as a potential abscission agent for table grapes: effects on pre-harvest abscission, fruit quality, and residue. Front Plant Sci 7:620

Fortes AM, Teixeira RT, Agudelo-Romero P (2015) Complex interplay of hormonal signals during grape berry ripening. Molecules 20:9326-9343

Gambetta GA, Matthews MA, Shaghasi TH, McElrone AJ, Castellarin SD (2010) Sugar and abscisic acid signaling orthologs are activated at the onset of ripening in grape. Planta 232:219-234

Gillaspy G, Bendavid H, Gruissem W (1993) Fruits-a developmental perspective. Plant Cell 5:1439-1451

Griesser M, Eder R, Besser S, Forneck A (2012) Berry shrivel of grapes in Austria-aspects of the physiological disorder with cultivar Zweigelt (Vitis vinifera L.). Sci Hortic 145:87-93. https ://doi.org/10.1016/j.scienta.2012.07.032
Griesser M, Martinez SC, Weidinger ML, Kandler W, Forneck A (2017) Challenging the potassium deficiency hypothesis for induction of the ripening disorder berry shrivel in grapevine. Sci Hortic 216:141-147

Griesser M, Martinez SC, Eitle MW, Warth B, Andre CM, Schuhmacher R, Forneck A (2018) The ripening disorder berry shrivel affects anthocyanin biosynthesis and sugar metabolism in Zweigelt grape berries. Planta 247:471-481

Haberer G, Kieber JJ (2002) Cytokinins. New insights into a classic phytohormone. Plant Physiol 128:354-362. https://doi. org/10.1104/pp.128.2.354

Hall GE, Bondada BR, Keller M (2011) Loss of rachis cell viability is associated with ripening disorders in grapes. J Exp Bot 62:11451153. https://doi.org/10.1093/Jxb/Erq355

Hellemans J, Mortier G, De Paepe A, Speleman F, Vandesompele J (2007) qBase relative quantification framework and software for management and automated analysis of real-time quantitative PCR data. Genome Biol 8(2):R19

Krasnow M, Matthews M, Shackel K (2008) Evidence for substantial maintenance of membrane integrity and cell viability in normally developing grape (Vitis vinifera $\mathrm{L}$.) berries throughout development. J Exp Bot 59:849-859. https://doi.org/10.1093/Jxb/Erm372

Krasnow MN, Matthews MA, Smith RJ, Benz J, Weber E, Shackel KA (2010) Distinctive symptoms differentiate four common types of berry shrivel disorder in grape. Calif Agric 64:155-159. https:// doi.org/10.3733/ca.v064n03p155

Krasnow MN, Matthews M, Shackel K (2012) Analysis of cell viability during ripening in healthy and SAD-affected grape berries. Am J Enol Viticult 63:460a

Kuhn N, Abello C, Godoy F, Delrot S, Arce-Johnson P (2014) Differential behavior within a grapevine cluster: decreased ethylenerelated gene expression dependent on auxin transport is correlated with low abscission of first developed berries. PLoS ONE 9(11):e111258

Kuhn N et al (2014) Berry ripening: recently heard through the grapevine. J Exp Bot 65:4543-4559. https://doi.org/10.1093/jxb/ert395

Kuiper D (1993) Sink strength-established and regulated by plantgrowth regulators. Plant Cell Environ 16:1025-1026

Kumar R, Khurana A, Sharma AK (2014) Role of plant hormones and their interplay in development and ripening of fleshy fruits. J Exp Bot 65:4561-4575

Ljung K (2013) Auxin metabolism and homeostasis during plant development. Development 140:943-950

Ma Y, Szostkiewicz I, Korte A, Moes D, Yang Y, Christmann A, Grill E (2009) Regulators of PP2C phosphatase activity function as abscisic acid sensors. Science 324:1064-1068

Massonnet $\mathrm{M}$ et al (2017) Ripening transcriptomic program in red and white grapevine varieties correlates with berry skin anthocyanin accumulation. Plant Physiol 174:2376-2396

Muday GK, Rahman A, Binder BM (2012) Auxin and ethylene: collaborators or competitors? Trends Plant Sci 17:181-195

Nishimura N et al (2010) PYR/PYL/RCAR family members are major in-vivo ABI1 protein phosphatase $2 \mathrm{C}$-interacting proteins in Arabidopsis. Plant J 61:290-299

Palumbo MC et al (2014) Integrated network analysis identifies fightclub nodes as a class of hubs encompassing key putative switch genes that induce major transcriptome reprogramming during grapevine development. Plant Cell 26:4617-4635

Pilati S et al (2017) Abscisic acid is a major regulator of grape berry ripening onset: new insights into ABA signaling network. Front Plant Sci 8:1-16

Reid KE, Olsson N, Schlosser J, Peng F, Lund ST (2006) An optimized grapevine RNA isolation procedure and statistical determination of reference genes for real-time RT-PCR 
during berry development. BMC Plant Biol 6:27. https://doi. org/10.1186/1471-2229-6-27

Savoi S et al (2017) Multi-omics and integrated network analyses reveal new insights into the systems relationships between metabolites, structural genes, and transcriptional regulators in developing grape berries (Vitis vinifera $\mathrm{L}$.) exposed to water deficit. Front Plant Sci 8:1124

Savoi S, Herrera JC, Forneck A, Griesser M (2019) Transcriptomics of the grape berry shrivel ripening disorder. Plant Mol Biol. https ://doi.org/10.1007/s11103-019-00859-1

Shin S, Lee J, Rudell D, Evans K, Zhu YM (2016) Transcriptional regulation of auxin metabolism and ethylene biosynthesis activation during apple (Malus x domestica) fruit maturation. J Plant Growth Regul 35:655-666

Sun LA, Zhang M, Ren J, Qi JX, Zhang GJ, Leng P (2010) Reciprocity between abscisic acid and ethylene at the onset of berry ripening and after harvest. BMC Plant Biol. https://doi. org/10.1186/1471-2229-10-257

Symons GM, Davies C, Shavrukov Y, Dry IB, Reid JB, Thomas MR (2006) Grapes on steroids. Brassinosteroids are involved in grape berry ripening. Plant Physiol 140:150-158. https://doi. org/10.1104/pp.105.070706

Tadiello A et al (2016) Interference with ethylene perception at receptor level sheds light on auxin and transcriptional circuits associated with the climacteric ripening of apple fruit (Malus $\mathrm{x}$ domestica Borkh.). Plant J 88:963-975

Uzquiza L, Martin P, Sievert JR, Arpaia ML, Fidelibus MW (2014) Methyl jasmonate and 1-aminocyclopropane-1-carboxylic acid interact to promote grape berry abscission. Am J Enol Viticult 65:504-509
Van de Poel B, Van Der Straeten D (2014) 1-Aminocyclopropane1-carboxylic acid (ACC) in plants: more than just the precursor of ethylene! Front Plant Sci 5:640

Wheeler S, Loveys B, Ford C, Davies C (2009) The relationship between the expression of abscisic acid biosynthesis genes, accumulation of abscisic acid and the promotion of Vitis vinifera $\mathrm{L}$. berry ripening by abscisic acid. Aust J Grape Wine Res 15:195204. https://doi.org/10.1111/j.1755-0238.2008.00045.x

Wybouw B, De Rybel B (2019) Cytokinin-a developing story. Trends Plant Sci 24:177-185. https://doi.org/10.1016/j.tplan ts.2018.10.012

Xu F, Xi ZM, Zhang H, Zhang CJ, Zhang ZW (2015) Brassinosteroids are involved in controlling sugar unloading in Vitis vinifera ' $\mathrm{Cab}$ ernet Sauvignon' berries during veraison. Plant Physiol Biochem 94:197-208

Zenoni S et al (2016) Disclosing the molecular basis of the postharvest life of berry in different grapevine genotypes. Plant Physiol 172:1821-1843

Zhang J, Peer WA (2017) Auxin homeostasis: the DAO of catabolism. J Exp Bot 68:3145-3154

Ziliotto F, Corso M, Rizzini FM, Rasori A, Botton A, Bonghi C (2012) Grape berry ripening delay induced by a pre-veraison NAA treatment is paralleled by a shift in the expression pattern of auxin- and ethylene-related genes. BMC Plant Biol 12(1):185

Publisher's Note Springer Nature remains neutral with regard to jurisdictional claims in published maps and institutional affiliations. 